\title{
RESEARCH
}

Open Access

\section{The proangiogenic effects of extracellular vesicles secreted by dental pulp stem cells derived from periodontally compromised teeth}

Huan Zhou' ${ }^{1}$ Xuan Li', Yuan Yin ${ }^{1}$, Xiao-Tao He ${ }^{1}$, Ying An ${ }^{1}$, Bei-Min Tian ${ }^{1}$, Yong-Long Hong ${ }^{2^{*}}$, Li-An Wu ${ }^{1 *}$ and Fa-Ming Chen ${ }^{1 *}$ (iD

\begin{abstract}
Background: Although dental pulp stem cells (DPSCs) isolated from periodontally compromised teeth (P-DPSCs) have been demonstrated to retain pluripotency and regenerative potential, their use as therapeutics remains largely unexplored. In this study, we investigated the proangiogenic effects of extracellular vesicles (EVs) secreted by PDPSCs using in vitro and in vivo testing models.

Methods: Patient-matched DPSCs derived from periodontally healthy teeth (H-DPSCs) were used as the control for P-DPSCs. Conditioned media (CMs) derived from H-DPSCs and P-DPSCs (H-CM and P-CM), CMs derived from both cell types pretreated with the EV secretion blocker GW4869 (H-GW and P-GW), and EVs secreted by H-DPSCs and PDPSCs (H-EVs and P-EVs) were prepared to test their proangiogenic effects on endothelial cells (ECs). Cell proliferation, migration, and tube formation were assessed using the Cell Counting Kit-8 (CCK-8), transwell/scratch wound healing, and Matrigel assays, respectively. Specifically, quantitative reverse transcriptase-polymerase chain reaction (qRT-PCR) and western blot analysis were used to examine the expression levels of angiogenesis-related genes/proteins in ECs in response to EV-based incubation. Finally, a full-thickness skin defect model was applied to test the effects of EVs on wound healing and new vessel formation.

(Continued on next page)
\end{abstract}

\footnotetext{
*Correspondence: ylhong93@163.com; lianwu@fmmu.edu.cn; cfmsunhh@fmmu.edu.cn

${ }^{2}$ Stomatology Center, Shenzhen Hospital of Southern Medical University, Shenzhen, Guangdong, People's Republic of China

'State Key Laboratory of Military Stomatology, National Clinical Research Center for Oral Diseases, School of Stomatology, Fourth Military Medical University, Xi'an 710032, Shaanxi, People's Republic of China
}

(c) The Author(s). 2020 Open Access This article is licensed under a Creative Commons Attribution 4.0 International License, which permits use, sharing, adaptation, distribution and reproduction in any medium or format, as long as you give appropriate credit to the original author(s) and the source, provide a link to the Creative Commons licence, and indicate if changes were made. The images or other third party material in this article are included in the article's Creative Commons licence, unless indicated otherwise in a credit line to the material. If material is not included in the article's Creative Commons licence and your intended use is not permitted by statutory regulation or exceeds the permitted use, you will need to obtain permission directly from the copyright holder. To view a copy of this licence, visit http://creativecommons.org/licenses/by/4.0/ The Creative Commons Public Domain Dedication waiver (http://creativecommons.org/publicdomain/zero/1.0/) applies to the data made available in this article, unless otherwise stated in a credit line to the data. 


\begin{abstract}
(Continued from previous page)
Results: Both $\mathrm{H}-\mathrm{CM}$ and $\mathrm{P}-\mathrm{CM}$ promoted $\mathrm{EC}$ angiogenesis, but the proangiogenic effects were compromised when ECs were incubated in H-GW and P-GW, wherein the EV secretion was blocked by pretreatment with GW4869. In EV-based incubations, although both $\mathrm{H}-\mathrm{EV}$ s and P-EVs were found to enhance the angiogenesis-related activities of ECs, P-EVs exerted a more robust potential to stimulate EC proliferation, migration, and tube formation. In addition, P-EVs led to higher expression levels of angiogenesis-related genes/proteins in ECs than H-EVs. Similarly, both P-EVs and $\mathrm{H}$-EVs were found to accelerate wound healing and promote vascularization across skin defects in mice, but wounds treated with P-EVs resulted in a quicker healing outcome and enhanced new vessel formation.
\end{abstract}

Conclusions: The findings of the present study provide additional evidence that P-DPSCs derived from periodontally diseased teeth represent a potential source of cells for research and therapeutic use. Particularly, the proangiogenic effects of P-EVs suggest that P-DPSCs may be used to promote new vessel formation in cellular therapy and regenerative medicine.

Keywords: Inflammation, Periodontitis, Dental pulp stem cells, Extracellular vesicles, Angiogenesis

\section{Background}

Dental pulp stem cells (DPSCs) are mesenchymal stem cell (MSC)-like populations that can be noninvasively harvested from pulp tissue of extracted human teeth; hence, these cells can be relatively easily obtained and are easy to access for use (reviewed in [1]). Due to their robust self-renewal capacity and pluripotency, DPSCs have paved the way for not only regenerating toothrelated tissues such as dental pulp [2] and the periodontium [3] but also repairing other types of tissue insults such as bone defects [4] and central nervous system damage [5]. Although accumulating evidence suggests that MSCs derived from bone marrow (BMMSCs), adipose tissue (ADSCs), and dental pulp (DPSCs) are all promising MSC types for regenerative purposes, DPSCs, at least in certain situations, were found to contain a higher percentage of stem/progenitor cells and to exhibit higher proliferation and osteogenic potential than donor-matched BMMSCs [6]. In addition, DPSCs have shown superior resistance to subculture, cryopreservation [7], and inflammation-induced senescence [8]. Taken together, these findings indicate that DPSCs are highly likely to maintain their multidifferentiation and regenerative potential within an inflamed microenvironment and thus would be suitable for use in combating inflammation-induced disease.

Although DPSCs can be isolated from exfoliated deciduous teeth, extracted wisdom teeth, and sometimes teeth extracted for orthodontic reasons [2, 5], we still cannot ensure that a patient will have a tooth available for cell isolation when cell therapy is needed. In this context, stem cells from allogeneic tissues might be an alternative choice, but the use of allogeneic MSCs adds additional safety and ethical concerns [9]. In view of this, scientists have sought to use DPSCs derived from inflamed pulp tissues, as these tissues are more easily accessible in clinics and often can be obtained from teeth with crown fractures [10], irreversible pulpitis [11-13], and periodontitis $[14,15]$. Particularly, pulp tissues within periodontally compromised teeth can be used for the production of DPSCs because these cells, to a certain degree, have been demonstrated to maintain their pluripotency and regenerative potential provided that pulp vitality is not totally lost $[14,15]$. Previously, we successfully isolated DPSCs from teeth that were extracted teeth due to severe periodontitis (P-DPSCs); their pluripotential capacity has been demonstrated using in vitro and in vivo models, suggesting that these traditionally discarded teeth may be an inexhaustible cell source for research and therapeutic purposes [14]. Although P-DPSCs are becoming relevant in cell production, how to use these cells for therapeutic purposes requires further exploration. Considering that the most direct use of DPSCs is pulp regeneration and that a prerequisite for cells to regenerate functional pulp is their proangiogenic effects for inducing vasculature formation, we herein investigated the angiogenic potential and vessel formation ability of P-DPSCs using in vitro and in vivo testing models.

Based on current understanding, the beneficial proangiogenic effects of stem cells in cellular therapy are most likely mediated via their paracrine mechanism (reviewed in [16]). It is now well recognized that stem cells can release an abundant mixture of cytokines, growth factors, chemokines, and extracellular vesicles (EVs), all of which have been demonstrated to contain functional elements that are useful for tissue regeneration. In particular, EVs within these secreted elements carry a complex cargo including but not limited to various mRNAs, microRNAs, and a spectrum of anti-apoptotic and proangiogenic factors; these agents are known to be the main mediators contributing to cell paracrine effects (reviewed in [17]). In this context, EVs derived from DPSCs have been demonstrated to trigger regeneration of dental pulp-like tissue [18] and indeed exhibit the capacity to promote angiogenesis in vitro and in vivo [19]. Therefore, the secreted elements contained within conditioned media 
(CMs) derived from P-DPSCs, particularly their secreted EVs, can be applied to investigate the proangiogenic effects of P-DPSCs.

In this study, we produced CMs using patientmatched DPSCs derived from periodontally healthy teeth (H-DPSCs) and P-DPSCs (H-CM and P-CM), CMs derived from both cells pretreated by GW4869 (EV secretion inhibited; $\mathrm{H}-\mathrm{GW}$ and P-GW), and isolated EVs secreted by H-DPSCs and P-DPSCs (H-EVs and P-EVs); these cellular materials were used in parallel to test their proangiogenic effects on endothelial cells (ECs). Finally, the ability of EVs (H-EVs and P-EVs) to improve wound healing and promote new vessel formation was tested in a full-thickness skin defect model. This is the first endeavor to explore the potential of using P-DPSCs for angiogenesis and new vessel formation in cellular therapy and regenerative medicine.

\section{Methods}

\section{Isolation and identification of H-DPSCs and P-DPSCs}

Patients visiting the Stomatological Hospital of Fourth Military Medical University (FMMU) who had (i) at least one tooth with full or partial pulp vitality that was to be extracted due to irreversible periodontitis and (ii) at least one other periodontally healthy tooth that was to be extracted due to nonfunctional or impacted reasons (often the third molars) were asked to donate their teeth for cell isolation and related research. The experimental protocol was approved by the Ethics Committee of the Stomatological Hospital of FMMU (201203), and informed consent was signed by all the subjects. Finally, 5 pairs of teeth were successfully obtained from 5 systemically healthy donors (male. 2; female. 3; age. 24 41 years); dental pulp tissues from periodontally healthy teeth $(n=5)$ and periodontitis teeth $(n=6)$ were used to isolate H-DPSCs and P-DPSCs in parallel according to our previously reported methods [14]. Briefly, the teeth were rinsed with adequate phosphate-buffered saline (PBS; Corning, NY, USA) before sawing to expose the pulp cavity. Then, the pulp tissues were removed from the teeth and cut into small pieces $(0.5-1 \mathrm{~cm}$ in length). After that, the tissue tips were immersed in digestive solution $(3 \mathrm{mg} / \mathrm{mL}$ collagenase type I; DIYIBio; Shanghai, China) and incubated for $1 \mathrm{~h}$ at $37^{\circ} \mathrm{C}$ (with vigorous shaking every $10 \mathrm{~min}$ ), followed by transfer into 12 -well plates (Corning, Lowell, MA, USA). The primary cells were cultured in $\alpha$-minimum essential medium $(\alpha-$ MEM; Gibco BRL, Grand Island, NY, USA) containing $10 \%$ fetal bovine serum (FBS; Sijiqing, Hangzhou, China) and $1 \%$ penicillin-G/streptomycin (Invitrogen, Carlsbad, CA, USA) at an atmosphere of $5 \% \mathrm{CO}_{2}$ at $37^{\circ} \mathrm{C}$ and passaged after reaching 80-90\% confluence. The limiting dilution technique was applied for DPSC purification. DPSCs at passages P3-P5 were characterized by a colony-forming assay, a proliferation assay, flow cytometry, and a multiple differentiation assay and then used for subsequent experiments.

For the colony-forming assay, DPSCs (P3) from both groups were seeded into 100-mm-diameter culture dishes (Corning) with complete $\alpha$-MEM at a density of $1 \times 10^{3}$ cells/dish. The culture medium was refreshed every 3 days. After incubation for 14 days, DPSCs were fixed with $4 \%$ paraformaldehyde (Servicebio, Wuhan, China) for $30 \mathrm{~min}$, stained with crystal violet (Heart, Xi'an, China) for $30 \mathrm{~min}$ and washed with distilled water three times. Then, the number of colonies was counted with an inverted microscope (Olympus, Tokyo, Japan), and only aggregates consisting of 50 or more cells were termed colonies.

A Cell Counting Kit-8 (CCK-8) assay was utilized to measure the proliferation ability of DPSCs. In brief, cells (P3) were seeded into 96-well plates (Corning) with complete $\alpha$-MEM at a density of $2 \times 10^{3}$ cells per well (four replicates per group). Ten microliters of CCK-8 reagent (Dojindo, Shanghai, China) was added to each well at a fixed time each day during the 7-day culture, and the cells were incubated for another $3 \mathrm{~h}$ at $37^{\circ} \mathrm{C}$. The optical density (OD) value was recorded at $450 \mathrm{~nm}$ by an Infinite M200 PRO microplate reader (TECAN, Männedorf, Switzerland).

A flow cytometry assay was applied to identify the cell immunophenotypes. Briefly, DPSCs (P3) were collected and washed twice with PBS containing 3\% FBS, and then, the cell suspension was divided into sterile Eppendorf tubes (Axygen, Tewksbury, MA, USA) and incubated with monoclonal antibodies against human CD90, CD105, CD146, CD34, CD45, or CD31 (all from eBioscience, San Diego, CA, USA) for $1 \mathrm{~h}$ at $4{ }^{\circ} \mathrm{C}$ in the dark. Cell suspensions incubated with PBS served as a negative control. Next, DPSCs were washed twice with PBS to remove excessive antibodies and then resuspended in $400 \mu \mathrm{L}$ PBS, followed by analysis with a Beckman Coulter Epics XL cytometer (Beckman Coulter, Fullerton, CA, USA).

To identify multiple differentiation potential, DPSCs (P4) were seeded into 6-well plates and cultured in complete $\alpha$-MEM at a density of $2 \times 10^{5}$ cells per well. After the DPSCs reached $80 \%$ confluence, their culture medium was changed to osteogenic differentiation medium, adipogenic differentiation medium, or chondrogenic differentiation medium (all from Cyagen, Guangzhou, China). After induction for 21 days (for osteogenic differentiation or adipogenic differentiation) or 28 days (for chondrogenic differentiation), differentiated cells were fixed with $4 \%$ paraformaldehyde and then separately subjected to Alizarin Red $S$ staining (for osteogenic differentiation), Oil Red O staining (for adipogenic differentiation), and Alcian blue staining (for chondrogenic differentiation). 
Conditioned medium (CM)-based incubation of ECs Preparation of CMs derived from H-DPSCs and P-DPSCs $\mathrm{H}$-DPSC- and P-DPSC-derived CMs were prepared in parallel based on the methods reported previously [20]. Briefly, DPSCs (H-DPSCs and P-DPSCs) were cultured with complete $\alpha-M E M$ and incubated until they reached approximately $90 \%$ confluence. Then, the cells were washed three times with PBS prior to culturing with serum-free $\alpha$-MEM media. After another incubation for $48 \mathrm{~h}$, the cell-derived supernatant was harvested, centrifuged at $3000 \times g$ for $3 \mathrm{~min}$ and $1500 \times g$ for $5 \mathrm{~min}$, filtered with $0.22-\mu \mathrm{m}$ filters (Millipore, Billerica, MA, USA) and stored at $-80{ }^{\circ} \mathrm{C}$ for further experiments. Cell-derived supernatants were also prepared from cells that were pretreated with culture media containing GW4869 (10 $\mu \mathrm{M}$; Sigma-Aldrich, St. Louis, USA) for $12 \mathrm{~h}$ before culturing in serum-free $\alpha$-MEM media, wherein GW4869 was used for the inhibition of EV secretion. Prior to use for EC culture, the supernatants derived from H-DPSCs, GW4869pretreated H-DPSCs, P-DPSCs, and GW4869-pretreated P-DPSCs were mixed with endothelial growth medium-2 (EGM-2; Lonza, Walkersville, MD, USA) at a ratio of 1:1 and termed H-CM, H-GW, P-CM, or P-GW. Cell proliferation, migration, and tube formation of ECs in response to incubation with various CMs were evaluated using established in vitro models, wherein a mixture of fresh $\alpha$ MEM medium and EGM-2 was used as a blank control for $\mathrm{H}-\mathrm{CM}$ and P-CM.

\section{Proliferation assay}

Human umbilical vein ECs were purchased from the American Type Culture Collection (ATCC; CRL-1730) and seeded in 96-well plates (Corning) at a concentration of $5 \times 10^{3}$ cells/well. Following culturing in various CMs (four replicates per CM), CCK-8-based cell proliferation assays (across a 5-day period) were performed as described previously in this manuscript.

\section{Cell migration assay}

Transwell and scratch wound healing assays were used to evaluate cell migration in response to various $\mathrm{CM}$ based incubations. For the transwell assay, ECs $\left(2 \times 10^{4}\right.$ cells/well) suspended in $100 \mu \mathrm{L}$ serum-free medium were seeded into the upper chamber of 24-well transwell culture plates $(8-\mu \mathrm{m}$ pore-sized filters; Corning), while $500 \mu \mathrm{L}$ CM (H-CM, P-CM, H-GW, or P-GW), or the established control medium for $\mathrm{H}-\mathrm{CM}$ and $\mathrm{P}-\mathrm{CM}$, was added to the lower chamber. After $8 \mathrm{~h}$ of incubation, nonmigrated cells on the upper surface of inserts were removed gently with a cotton swab, and then, the cells that migrated to the lower surface of membranes were fixed with $4 \%$ paraformaldehyde prior to staining with crystal violet (Heart) for $30 \mathrm{~min}$. Images were captured, and the number of migrated cells was counted in five randomly selected fields per well. For the scratch wound healing assay, ECs $\left(4 \times 10^{5}\right.$ cells/well $)$ were seeded into 6-well plates (Corning). After the cells reached $90 \%$ confluence, one scratch was made in each well with a sterile $1 \mathrm{~mL}$ pipette tip, and then, the floating cells were removed by washing twice with PBS. After that, ECs were exposed to the various CMs. Scratch images were obtained at baseline $(0 \mathrm{~h})$ and at different observation time points $(12 \mathrm{~h} ; 24 \mathrm{~h})$, and the outcomes were analyzed with ImageJ software.

\section{Tube formation assay}

A Matrigel tube formation assay was conducted to investigate network formation of ECs by the method described previously [21]. In brief, 96-well plates were coated with $50 \mu \mathrm{L}$ cold Matrigel (Corning; \#356234) and incubated at $37^{\circ} \mathrm{C}$ for $30 \mathrm{~min}$. Then, $110 \mu \mathrm{L}$ different CMs containing $2 \times 10^{4}$ ECs were added to each well. After incubation for $6 \mathrm{~h}$, the images of tube formation were observed and photographed. Indicators of tube formation ability, including covered area, total branching points, total tube length, and total loops, were analyzed with ImageJ software in five randomly chosen fields.

\section{Production of EVs derived from H-DPSCs and P-DPSCs $(\mathrm{H}-$ EVs and P-EVs)}

For extraction of EVs, the medium of H-DPSCs or PDPSCs was collected after $48 \mathrm{~h}$ of culture, and the centrifugation steps were performed as described previously [22]. Briefly, the harvested medium was centrifuged at $300 \times g$ for $10 \mathrm{~min}$ and $2000 \times g$ for $10 \mathrm{~min}$ to eliminate cells. Then, the obtained supernatant was centrifuged at $10,000 \times g$ for $30 \mathrm{~min}$ to eliminate cellular debris. After that, the following supernatant was centrifuged at $100,000 \times g$ for $70 \mathrm{~min}$ and additionally washed with PBS at $100,000 \times g$ for $70 \mathrm{~min}$ (Ultracentrifuge, Beckman Coulter). Finally, the supernatant was discarded, and EV pellets were resuspended in PBS for further experiments or cryopreserved at $80{ }^{\circ} \mathrm{C}$. The morphologies of EVs were observed with transmission electron microscopy (Hitachi, Tokyo, Japan), the concentration and size of EVs were measured and analyzed with nanoparticle tracking analysis (NanoSight 300; Malvern Instruments, Malvern, UK), and biomarker proteins for EVs were detected by the western blot assay (see the "Western blot analysis" section). To examine the incorporation of EVs, H-EVs and P-EVs were labeled with PKH67 (Sigma-Aldrich, St. Louis, MO, USA) according to the protocol. Briefly, EVs were resuspended in diluent $\mathrm{C}$, mixed with PKH67, and incubated for $4 \mathrm{~min}$. Then, 5\% bovine serum albumin (BSA; heart) was added to neutralize the reaction. Afterwards, the labeled EVs were washed in PBS at $100,000 \times g$ for $70 \mathrm{~min}$ and then 
incubated with ECs at $37^{\circ} \mathrm{C}$ for $4 \mathrm{~h} .4$ ',6-diamidino-2-phenylindole (DAPI; Heart) was used to label the nuclei. Images of EV uptake were obtained with a confocal imaging system (Carl Zeiss, Oberkochen, Germany).

\section{EV-based incubation of ECs}

Similar to CM-based incubation, cell proliferation, migration, and tube formation of ECs in response to incubation with various EVs were determined, wherein the medium was changed to endothelial basal medium (EBM-2; Lonza) containing 5\% FBS in the presence of $100 \mu \mathrm{g} / \mathrm{mL} \mathrm{H}$-EVs (H-EVs group) or $100 \mu \mathrm{g} / \mathrm{mL} \mathrm{P-EVs}$ (P-EVs group); the same amount of PBS was used as a control (control group). Quantitative real-time PCR (qRT-PCR) and western blot analysis were applied to evaluate the cellular responses at the gene and protein levels.

\section{qRT-PCR analysis}

For gene expression analysis, cell samples were washed twice with PBS. After that, total RNA was extracted with a MiniBEST Universal RNA Extraction Kit (Takara, Tokyo, Japan) and then reverse transcribed to cDNA using the PrimeScript $^{\text {tw }}$ RT reagent Kit (Takara). qRT-PCR analysis with TB Green ${ }^{\text {Tw }}$ Premix Ex Taq ${ }^{\text {Tim }}$ II (Takara) was conducted and analyzed using a Real-Time PCR Detection System (Bio-Rad, Hercules, CA, USA). $\beta$-Actin was employed to normalize the levels of the genes of interest. Primer sequences used for qRT-PCR were as follows: $\beta$ actin: forward, 5 '-TGGCACCCAGCACAATGAA-3', and reverse, 5' -CTAAGTCATAGTCCGCCTAGAAGCA-3'; h-VEGF: forward, 5' - CATCCAATCGAGACCCTGGTG3', and reverse, 5'-TTGGTGAGGTTTGATCCGCATA3'; and h-AngII: forward, 5'-GCTGAAGTATTCAA ATCAGGACACA-3', and reverse, 5'-ATCAACGCTG CCATCCTCA-3.

\section{Western blot analysis}

The protein extracts of cells (or EVs, see the "Production of EVs derived from H-DPSCs and P-DPSCs (H-EVs and P-EVs)" section) were subjected to sodium dodecyl sulfate-polyacrylamide gel electrophoresis (SDS-PAGE) and transferred to PVDF membranes (Millipore, Billerica, MA, USA). Then, the membranes were blocked in 5\% nonfat milk in Tris-buffered saline Tween-20 (TBST, Heart). After incubating with primary antibodies including anti- $\beta$-actin (1:1000; Proteintech, Rosemont, USA; \#60008-1-lg), anti-ALIX (1:1000; Cell Signaling Technology, Danvers, MA, USA; \#2171), anti-HSP-70 (1:1000; Cell Signaling Technology; \#4876), anti-CD9 (1:1000; Cell Signaling Technology; \#13174), anti-CD81 (1:1000; Abcam, Cambridge, Britain; ab109201), anti-VEGF(1: 1000; Abcam; ab46154), and anti-AngII (1:100; Santa Cruz, CA, USA; sc-74,403) at $4{ }^{\circ} \mathrm{C}$ overnight, the membranes were washed three times with TBST. Then, the cells were incubated with horseradish peroxidaseconjugated secondary antibodies (1:5000, Proteintech; SA00001-1 or SA00001-2) for $2 \mathrm{~h}$ at room temperature. Subsequently, blots were detected using chemiluminescent detection reagent (Zeta Life, CA, USA), and the protein bands were analyzed with ImageJ software. $\beta$ actin was employed as the housekeeping gene for internal normalization.

\section{Use of EVs in a skin wound healing model Mouse skin wound model and treatments}

Thirty male C57BL/6 mice (8 weeks old, weighing 20$25 \mathrm{~g}$; purchased from the Animal Research Committee of FMMU) were used in this study, and all procedures were approved by the Animal Research Committee of FMMU. A full-thickness excisional skin wound was created on the dorsum of each mouse as previously reported [23]. Then, the mice were randomly divided into three groups (10 mice per group): an H-EV group, a P$\mathrm{EV}$ group, and a control group. Mice in each group were designated to receive subcutaneous injection of either H-EVs $(200 \mu \mathrm{g}$ in $100 \mu \mathrm{L}$ PBS $)$, P-EVs $(200 \mu \mathrm{g}$ in $100 \mu \mathrm{L}$ $\mathrm{PBS}$ ) or $100 \mu \mathrm{L}$ of PBS only (4 sites around the wounds; $25 \mu \mathrm{L}$ per site). To evaluate the rate of wound healing, wounds were photographed and measured with a metric ruler at baseline and 4, 9, and 14 days after treatment, and the wound area was analyzed with ImageJ software. The undersurface of the skin was observed by a microscope (Olympus) to detect newly formed vessels on day 14 after the operation.

\section{Histological and immunofluorescence analysis}

Vascularization is essential to wound healing and tissue repair (reviewed in [24]). Thus, histological analysis and immunohistochemistry staining for angiogenesis markers were used to evaluate wound closure as well as newly formed vessels. Two weeks after surgery, mice were sacrificed, and the skin specimens were harvested and analyzed according to the methods reported previously [23]. Briefly, the collected skin tissues (including the wound healing and surrounding healthy skin) were fixed with $4 \%$ paraformaldehyde solution, dehydrated with ethanol, and embedded in paraffin. Then, the specimens were cut into $10-\mu \mathrm{m}$-thick sections and subjected to hematoxylin and eosin (H\&E) staining. In addition, immunofluorescence staining for VEGF and CD31 was performed to estimate the newly formed capillaries, as previously reported [25]. In brief, the obtained samples were fixed with $4 \%$ paraformaldehyde overnight at $4{ }^{\circ} \mathrm{C}$, dehydrated in $30 \%$ sucrose solution, and then embedded. Then, the 10$\mu \mathrm{m}$-thick sections were incubated with primary antibodies, including anti-VEGF (1:100, Abcam; ab2349) 
A

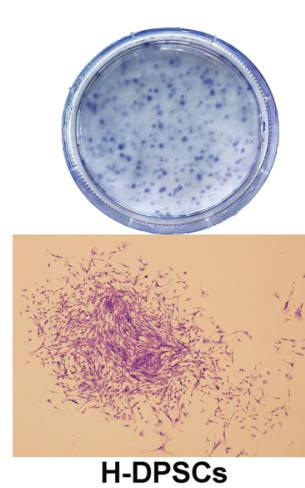

H-DPSCs

C
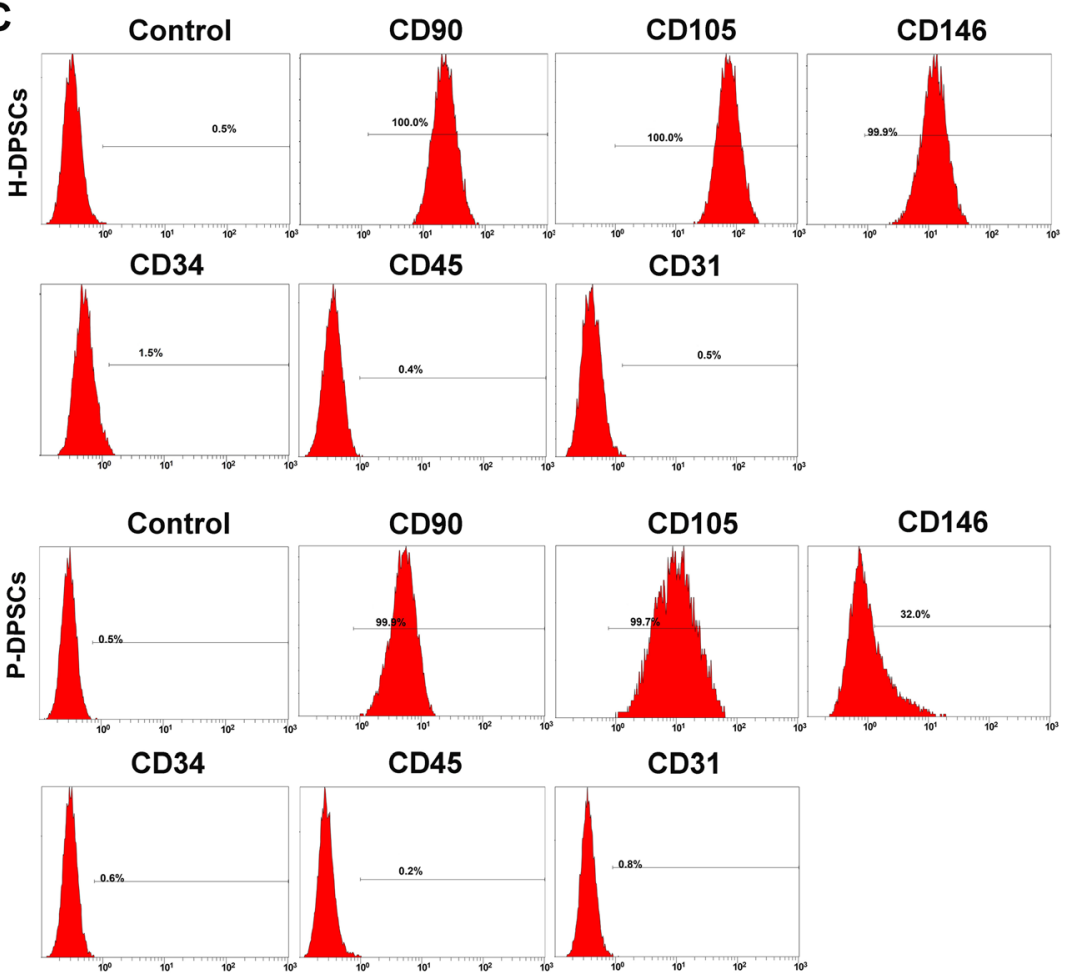

D

Osteogenic
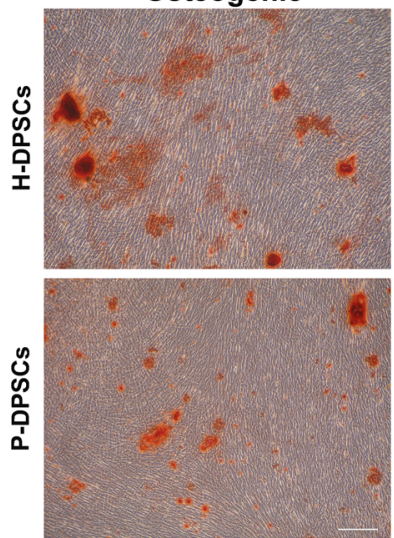
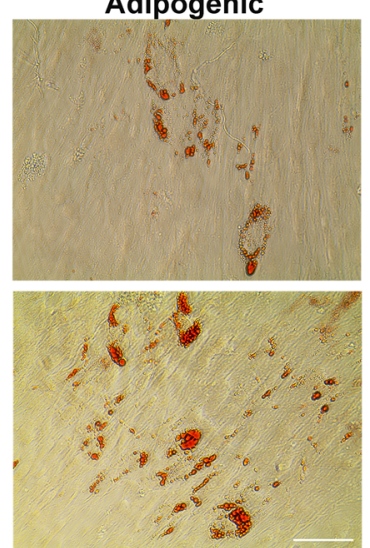

$\rightarrow$ H-DPSCs

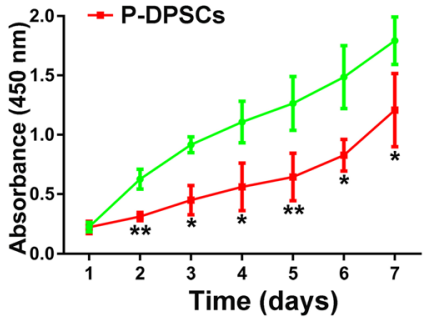

CD105 CD146

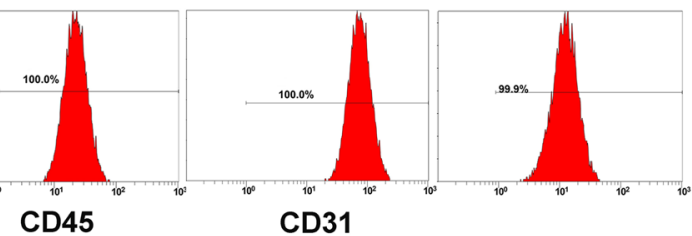

Chondrogenic

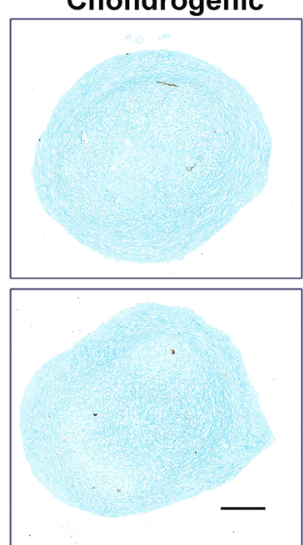

Fig. 1 (See legend on next page.) 
(See figure on previous page.)

Fig. 1 Isolation and identification of H-DPSCs and P-DPSCs. a Representative images of colony units in a general view (first row of images) and a single colony of H-DPSCs and P-DPSCs observed by microscopy (second row of images; scale bar, $200 \mu \mathrm{m}$ ). $\mathbf{b}$ Proliferative activity of H-DPSCs and P-DPSCs evaluated by CCK-8 assay $\left(n=3 ;{ }^{*} P<0.05,{ }^{* *} P<0.01\right.$ vs. the H-DPSC group). c Surface markers of H-DPSCs and P-DPSCs assayed by flow cytometry. d Multiple differentiation potentials of H-DPSCs and P-DPSCs: Alizarin Red S staining for osteogenic differentiation (left; scale bar, $200 \mu \mathrm{m}$ ), Oil Red O staining for adipogenic differentiation (middle; scale bar, $200 \mu \mathrm{m}$ ), and Alcian blue staining for chondrogenic differentiation (right; scale bar, $200 \mu \mathrm{m}$ )

and anti-CD31 (1:100, Abcam; ab9498), overnight at $4{ }^{\circ} \mathrm{C}$ and then incubated with secondary antibodies (1: 300 or 1:400, Servicebio; GB21303 or GB25303) for 1 $\mathrm{h}$ at room temperature. Images were captured and analyzed with Image-Pro Plus 6 software.

\section{Statistical analysis}

All assays were performed at least three times independently, and the data are presented as the mean \pm standard deviation (S.D.). Differences between groups were assessed by the paired $t$ test (for two-group comparisons) and one-way analysis of variance (ANOVA) followed by Tukey's posttest (for multiple groups). A level of $p<0.05$ was judged to be statistically significant.

\section{Results}

\section{Characterization of H-DPSCs and P-DPSCs}

$\mathrm{H}$-DPSCs and P-DPSCs were successfully isolated from pulp tissues of 11 teeth (periodontally healthy teeth, 5; periodontitis teeth, 6). Five teeth belonging to 2 donors were excluded due to culture failure (if cell isolation failed for one tooth, the participant was excluded). Almost all of the cells initially grew from pulp tissues after incubation for 7 days (Fig. S1a) and exhibited clone-like growth as well as a spindle-shaped appearance (Fig. S1b). It was apparent that DPSCs in both groups presented colony-forming ability (Fig. 1a). In terms of the CCK-8 assay, H-DPSCs displayed a significantly higher proliferative rate than P-DPSCs $(P<0.05$ or $P<0.01$; Fig. $1 \mathrm{~b})$. Flow cytometry assays were conducted to detect the surface markers of DPSCs. Notably, both P-DPSCs and H-DPSCs were positive for MSC markers, including CD90, CD105, and CD146, and negative for hematopoietic cell markers, such as CD31, CD34, and CD45 (Fig. 1c). In addition, both P-DPSCs and H-DPSCs could readily be induced to differentiate into osteogenic, adipogenic, and chondrogenic lineages when cultured in their respective differentiation media, as evidenced by Alizarin Red $S$ staining, Oil Red $\mathrm{O}$ staining, and Alcian blue staining, respectively (Fig. 1d).

Cellular responses of ECs to incubation with various $\mathrm{CMs}$ $P$-CM enhanced the angiogenic activities of ECs in vitro

It is well recognized that paracrine activity contributes considerably to MSC-based therapy [26]. To analyze the paracrine effects of DPSCs, ECs were exposed to $\mathrm{H}-\mathrm{CM}$ and $\mathrm{P}-\mathrm{CM}$, and angiogenic activities related to proliferation, migration, and tube formation were analyzed. As shown in Fig. 2a, both $\mathrm{H}-\mathrm{CM}$ and $\mathrm{P}-\mathrm{CM}$ significantly enhanced EC proliferation compared with the control $(P<0.05$ or $P<0.01)$; additionally, the proliferation rate was higher in the $\mathrm{P}-\mathrm{CM}$ group than in the $\mathrm{H}-\mathrm{CM}$ group $(P<0.05$ or $P<0.01)$. Similar trends were observed in the transwell migration assay $(P<0.05$ or $P<0.01$; Fig. $2 \mathrm{~b}, \mathrm{c})$ and scratch wound healing assay $(P<0.05$ or $P<$ 0.01; Fig. $2 \mathrm{~d}, \mathrm{e})$. In addition, tube formation assays revealed that incubation with either $\mathrm{P}-\mathrm{CM}$ or $\mathrm{H}-\mathrm{CM}$ could promote angiogenesis when compared with that in the control group (Fig. 2f). With the exception of the covered area $(P>0.05)$, ECs incubated with P-CM demonstrated a stronger tube formation ability than those incubated with $\mathrm{H}-\mathrm{CM}$ in terms of quantitative analyses of the total branching points $(P<0.01)$, total loop numbers $(P<0.05)$, and total tube length $(P<0.01)$ (Fig. $2 \mathrm{~g})$.

\section{Inhibition of EV secretion impaired CM-mediated angiogenesis}

Currently, EVs are considered to be a vital paracrine product of MSCs [27]. If EVs contribute to the proangiogenic effects of DPSCs, then inhibition of EV release would be expected to abolish or reduce the function. To verify this hypothesis, we pretreated H-DPSCs and P-DPSCs with GW4869 (a reversible blocker of neutral sphingomyelinase that controls EV secretion) [28]. It was observed that $10 \mu \mathrm{M}$ GW4869 successfully decreased EV release by H-DPSCs and P-DPSCs (Fig. S2b) without affecting cell growth (Fig. S2a). Indeed, the increased proliferation trend of ECs exposed to H-CM and P-CM was partially inhibited by GW4869 pretreatment, as a CCK- 8 assay revealed that both cells exposed to $\mathrm{H}-\mathrm{GW}$ and P-GW had a lower proliferation rate than cells exposed to H-CM or P-CM $(P<0.05$ or $P<0.01$; Fig. $3 a)$, respectively. In addition, the increased migration abilities of ECs stimulated by $\mathrm{H}-\mathrm{CM}$ and $\mathrm{P}-\mathrm{CM}$ were also attenuated by GW4869 pretreatment, with fewer migrated cells quantified by transwell migration $(P<$ 0.05 ; Fig. $3 \mathrm{~b}, \mathrm{c})$ and larger scratch wounds in the scratch wound assay $(P<0.05$ or $P<0.01$; Fig. $3 \mathrm{~d}$, e). 


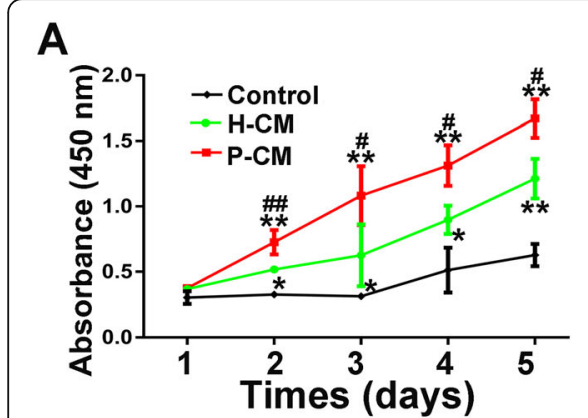

B

C

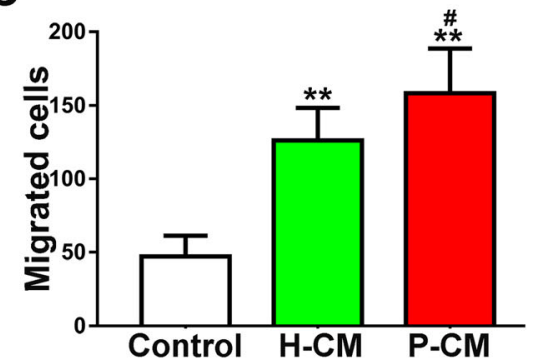

E

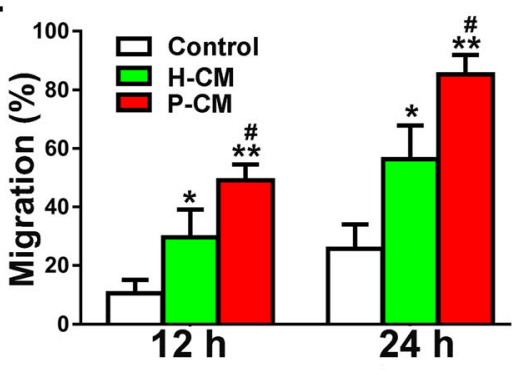

D $\quad 0 \mathrm{~h}$

$12 \mathrm{~h}$

$24 \mathrm{~h}$
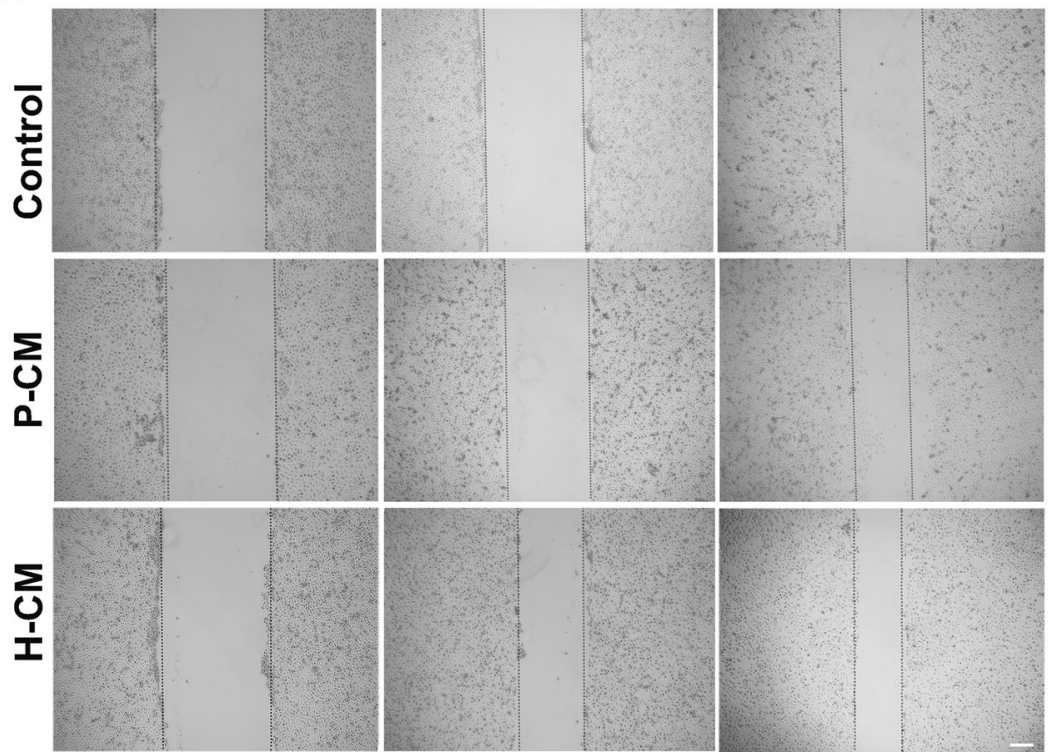

$\mathbf{F}$

Control

$\mathrm{H}-\mathrm{CM}$

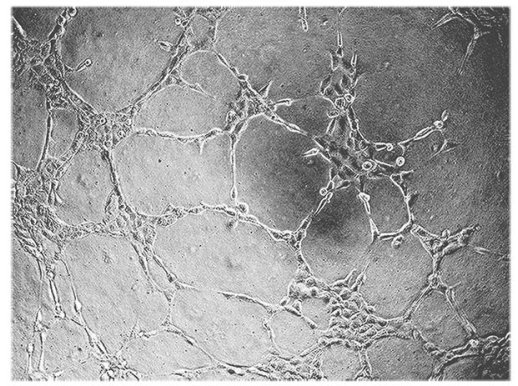

G

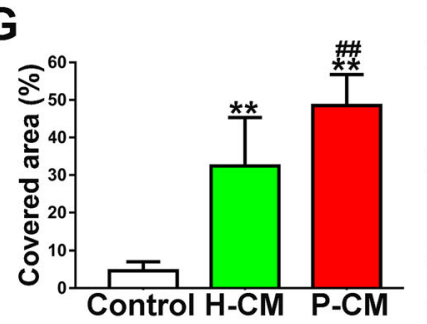

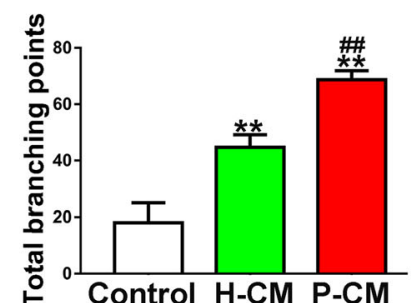

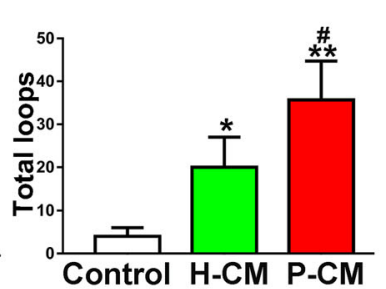

P-CM
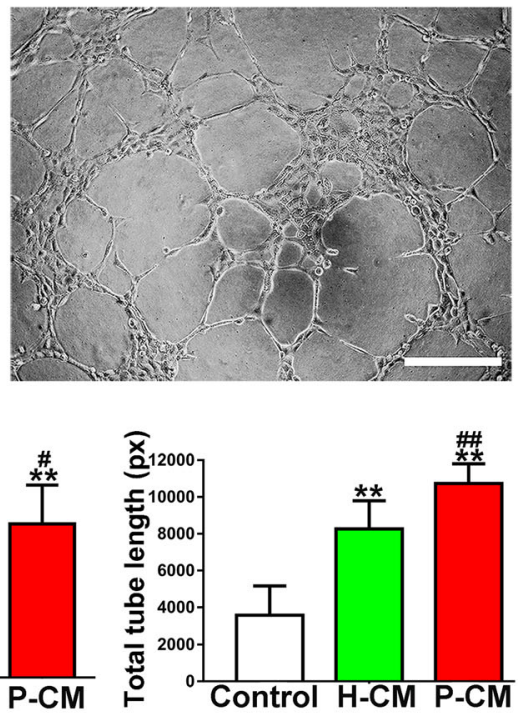

Fig. 2 (See legend on next page.) 
(See figure on previous page.)

Fig. $2 \mathrm{H}-\mathrm{CM}$ and P-CM enhanced the angiogenic activities of ECs. a The proliferation of ECs exposed to H-CM and P-CM was tested by CCK-8 assay $(n=3)$. $\mathbf{b}$ The migration of ECs stimulated by H-CM and P-CM was detected by transwell assay (scale bar, $100 \mu \mathrm{m})$. c Quantitative analysis of the migrated cells in $\mathbf{b}(n=5)$. $\mathbf{d}$ Representative images of the scratch wound assay of ECs treated with H-CM and P-CM (scale bar, $200 \mu \mathrm{m})$. $\mathbf{e}$ Quantitative analysis of the migration rates in $\mathbf{d}(n=5)$. $\mathbf{f}$ Representative images of the tube formation assay in ECs treated with $\mathrm{H}-\mathrm{CM}$ and P-CM (scale bar, $200 \mu \mathrm{m})$. g Quantitative analyses of the covered area, total branching points, total loops, and total tube length in $\mathbf{f}(n=3)$. ${ }^{*} P<0.05$, ${ }^{* *} P<0.01$ vs. the control group; ${ }^{\#} P<0.05,{ }^{\# \#} P<0.01$ vs. the $\mathrm{H}-\mathrm{CM}$ group

Notably, H-GW and I-GW partially reversed the enhanced tube formation trend by $\mathrm{H}-\mathrm{CM}$ and $\mathrm{P}-\mathrm{CM}$, as quantified by the capillary structure formations of ECs $(P<0.05$ or $P<0.01$; Fig. $3 f, g)$.

\section{Identification of H-EVs and P-EVs}

Given that EVs played a crucial role in the paracrine function-mediated angiogenesis of DPSCs, H-EVs and PEVs were isolated for further study. As expected, nanoparticles purified from the supernatants were both bilayer membrane vesicles (Fig. 4a), coinciding with the general characteristics of EVs, and nanoparticle analysis showed that the EV diameters mostly ranged from 30 to $200 \mathrm{~nm}$ in both groups (Fig. 4b). Additionally, western blot analysis revealed that both $\mathrm{H}$-EVs and P-EVs expressed EV molecular markers, including ALIX, HSP70, CD9, and CD81 (Fig. 4c), further confirming their EV identity. Subsequently, to confirm cellular EV uptake, PKH67-labeled H-EVs and P-EVs were cocultured with ECs. After incubating for $4 \mathrm{~h}$, ECs were observed to stain positive for PKH67 in both groups, confirming EV uptake (Fig. 4d).

\section{Cellular responses of ECs to H-EV/P-EV-based incubation $P$-EVs promoted the proliferation and migration of ECS in vitro}

ECs were treated with P-EVs, H-EVs, or an equal volume of PBS for a sequence of functional assays, including proliferation and migration, which are two critical steps of angiogenesis. The results obtained from the CCK-8 assay showed that the proliferation rate of ECs was markedly elevated in response to EV stimulation relative to the control group $(P<0.05$ or $P<0.01$; Fig. 5a), and notably, ECs exhibited a stronger proliferative rate by P-EVs than H-EVs $(P<0.05$; Fig. 5a). The migrationpromoting effect was determined by a scratch wound healing assay and a transwell assay. The results obtained from the transwell assay revealed that there was no difference between the two EV groups $(P>0.05$; Fig. $5 \mathrm{~b}, \mathrm{c})$, although both of them demonstrated a stronger migration-promoting ability than the control group $(P<0.01$; Fig. 5b, c). In the scratch wound healing assay, both H-EVs and P-EVs remarkably upregulated the motility of ECs compared with that in the control group $(P<0.01 ;$ Fig. $5 \mathrm{~d}, \mathrm{e})$, and P-EVs exhibited a stronger migration ability than H-EVs $(P<$ 0.05; Fig. 5d, e).

\section{P-EVs potentiated the angiogenic activities of ECs in vitro}

Furthermore, we evaluated the effect of EVs on angiogenic activities of ECs. Compared with the control group, both EV treatments significantly enhanced the angiogenic activities of ECs $(P<0.01$; Fig. 6a, b). Moreover, compared with those treated with H-EVs, ECs treated with P-EVs showed a higher number of capillary-like structures, and quantitative measurements revealed that the total branching points, total loop numbers, and total tube length were all significantly increased $(P<0.01$; Fig. 6b), although the covered area was not $(P>0.05$; Fig. 6b). qRT-PCR and western blot analysis were performed to detect the expression levels of angiogenesis-related genes and proteins (VEGF and AngII). The obtained results demonstrated that both groups of EVs exhibited upregulated angiogenesisrelated gene expression compared with that in the control group $(P<0.05$ or $P<0.01$; Fig. $6 \mathrm{c})$; moreover, gene expression in the $\mathrm{P}-\mathrm{EV}$ group was higher than that in the H-EV group $(P<0.05$ or $P<0.01$; Fig. $6 \mathrm{c})$. Furthermore, ECs in the P-EV group secreted more VEGF and AngII proteins than ECs in the $\mathrm{H}-\mathrm{EV}$ and control groups $(P<0.05$ or $P<0.01$; Fig. $6 \mathrm{~d}$, e).

\section{Effects of H-EVs and P-EVs on wound healing $P$-EVs accelerated cutaneous wound healing in mice}

A rat full-thickness defect model was created to assess the effects of EVs on wound healing. All the mice were maintained under specific pathogen-free (SPF) conditions, and none of the mice showed any signs of discomfort/disability following EV administration in the present study. Wound closure was markedly accelerated by EV treatment, illustrated by smaller wound areas measured at days 3,9 , and 14 postwounding compared with those in the control group $(P<0.05$ or $P<0.01$; Fig. $7 \mathrm{a}, \mathrm{b})$, and as expected, the rate of wound closure in the $\mathrm{P}-\mathrm{EV}$ group was faster than that in the $\mathrm{H}-\mathrm{EV}$ group $(P<0.05$ or $P<0.01$; Fig. $7 \mathrm{~b})$. In addition, the wounds treated with P-EVs had almost entirely closed by day 14 after the operation, whereas relatively larger scar areas remained detectable in the control group (Fig. 7a). H\&E staining revealed that transplantation with both types of EVs reduced the scar formation of 

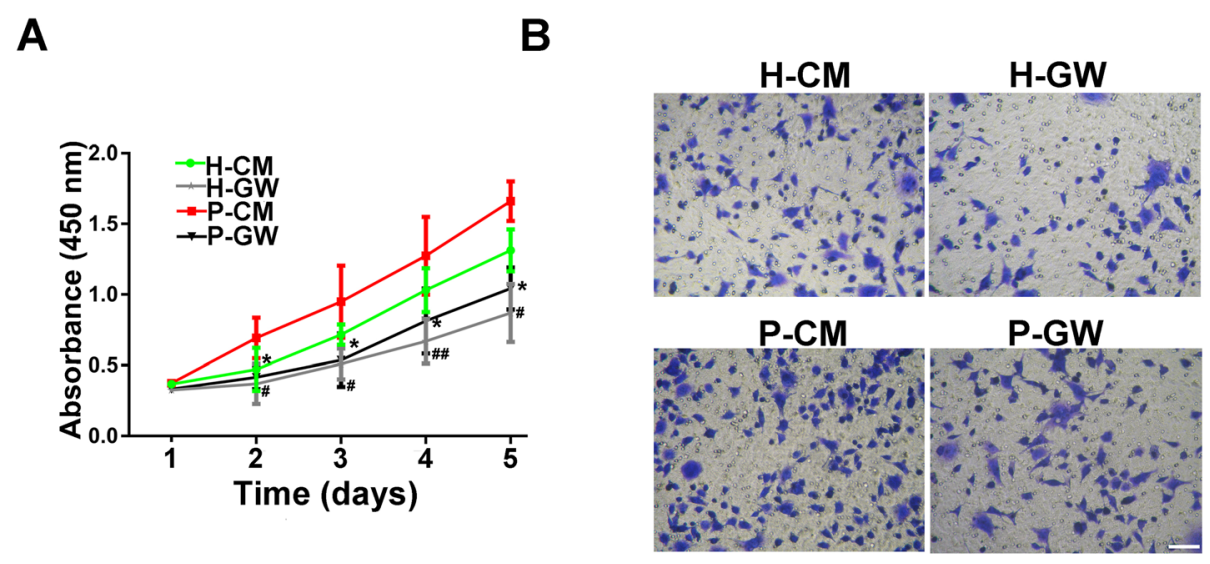

C

D

$\mathrm{oh}$

$12 \mathrm{~h}$

$24 \mathrm{~h}$
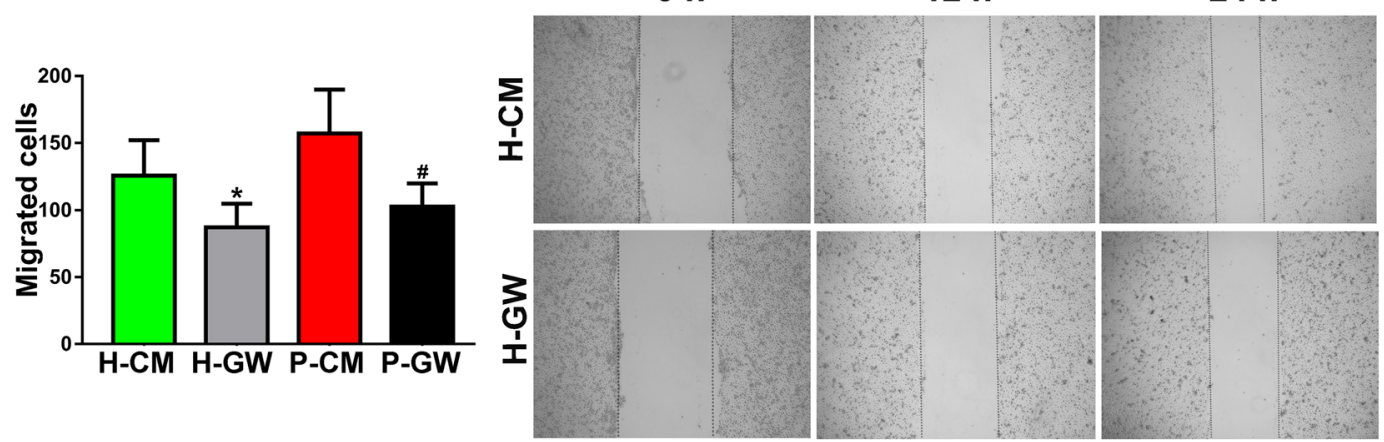

E

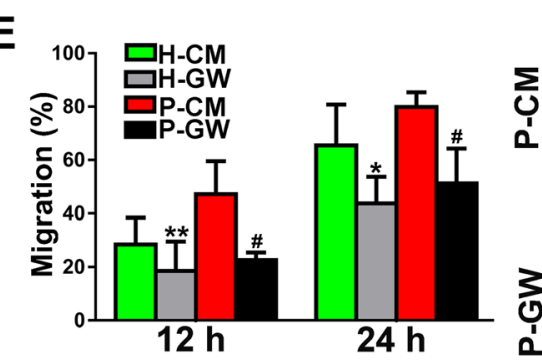

$\mathbf{F}$

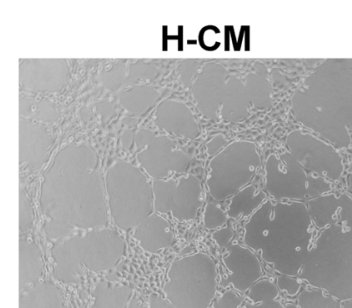

H-GW

P-CM

P-GW
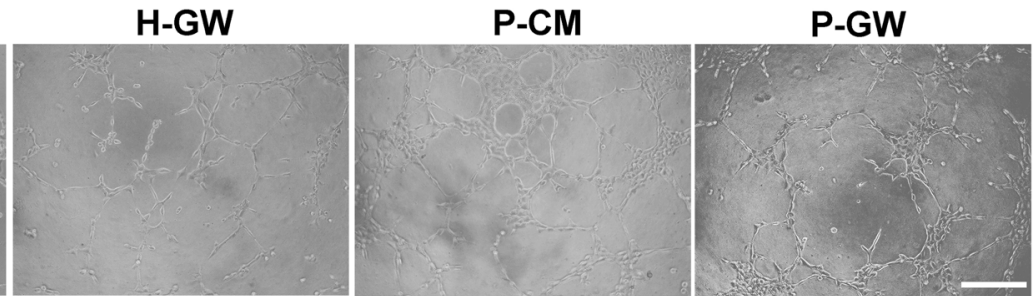

G

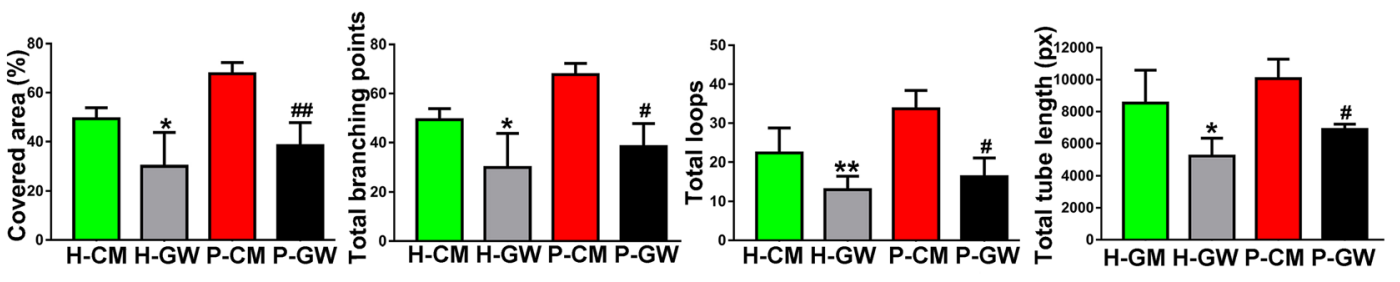

Fig. 3 (See legend on next page.) 
(See figure on previous page.)

Fig. 3 EVs play a pivotal role in CM-mediated angiogenesis. a The proliferation of ECs exposed to H-CM, H-GW, P-CM, and P-GW was tested by CCK-8 assay $(n=3)$. b The migration of ECs stimulated by H-CM, H-GW, P-CM, and P-GW was detected by transwell assay (scale bar, $100 \mu$ m). $\mathbf{c}$ Quantitative analysis of the migrated cells in $\mathbf{b}(n=3)$. $\mathbf{d}$ Representative images of the scratch wound assay of ECs treated with $\mathrm{H}-\mathrm{CM}, \mathrm{H}-\mathrm{GW}, \mathrm{P}-$ CM, and P-GW (scale bar, $200 \mu \mathrm{m}$ ). e Quantitative analysis of the migration rates in $\mathbf{d}(n=3)$. $\mathbf{f}$ Representative images of tube formation in ECs treated with H-CM, H-GW, P-CM, and P-GW (scale bar, $200 \mu \mathrm{m})$. g Quantitative analyses of the covered area, total branching points, total loops, and total tube length in $\mathbf{f}(n=3) .{ }^{*} P<0.05,{ }^{* *} P<0.01$ vs. the $\mathrm{H}-\mathrm{CM}$ group; ${ }^{\#} P<0.05$, ${ }^{\# \#} P<0.01$ vs. the $\mathrm{P}-\mathrm{CM}$ group

wounds compared with the control group at day 14 after treatment $(P<0.01$; Fig. $7 \mathrm{c}, \mathrm{d})$, and quantitative measurements confirmed that P-EV-treated wounds had a lower level of scar formation than H-EV-treated wounds $(P<0.05$; Fig. $7 \mathrm{~d})$.

\section{P-EVs enhanced vessel formation in the wound sites of mice} Skin images from the undersurface revealed that both EV-treated groups exhibited more newly formed microvessels than control wounds at day 14 postwounding (Fig. 8a). Immunofluorescence staining for endothelial markers, including CD31 and VEGF, was also performed to quantify the density of new blood vessels. Representative images of CD31 staining consistently demonstrated that abundant blood vessels appeared in the wounds treated with EVs, whereas the capillary density was significantly lower in the control group
$(P<0.01$; Fig. 8b, c). Quantitative analysis revealed that the proangiogenic effect of P-EVs was stronger than that of H-EVs $(P<0.01$; Fig. $8 \mathrm{c})$. Similarly, more extensive blood vessel staining for VEGF was observed in the P-EV group than in the $\mathrm{H}-\mathrm{EV}$ and control groups $(P<0.01$, Fig. 8 d, e).

\section{Discussion}

The regeneration of dental pulp tissue must incorporate a bioengineering strategy to promote cell chemotaxis and angiogenesis and, hence, fast vascularization of newly formed tissue [29]. Only when new vessels and vasculature can provide oxygen and nutrients to the stem cells involved in the reparative process can functional tissue regeneration be finally successful. However, the ability of current strategies to promote angiogenesis/vasculogenesis to form vascularized dental
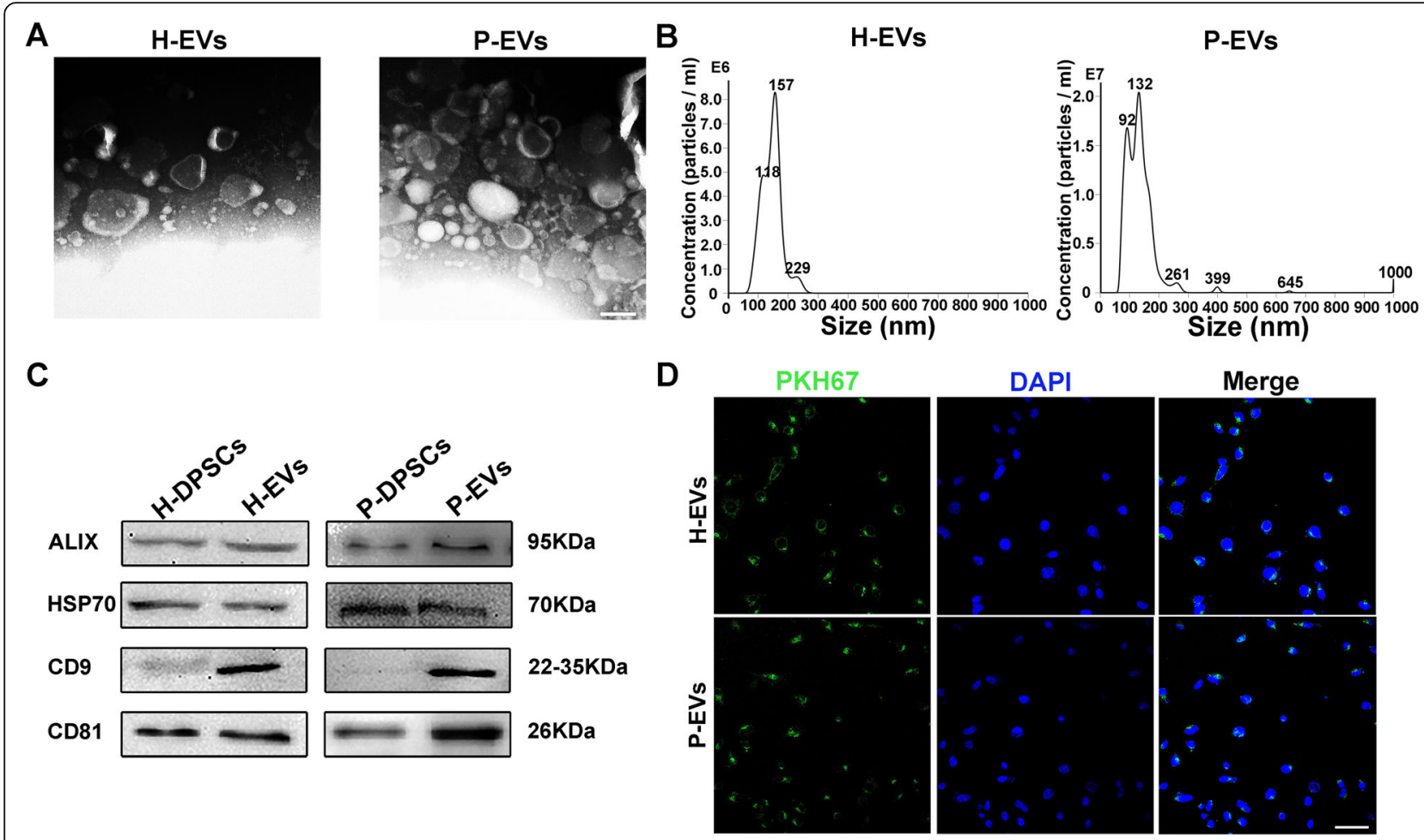

Fig. 4 Identification and internalization of H-EVs and P-EVs. a Morphology of EVs under transmission electron microscopy (scale bar, $100 \mathrm{~nm}$ ). b The size and concentration of EVs were identified by nanoparticle analysis. c EV surface markers of ALIX, HSP70, CD9, and CD81 detected by western blot. d Fluorescence microscopy analysis of PKH67-labeled EVs (green) internalized by ECs. Nuclei were stained with DAPI (blue) for counterstaining (scale bar, $50 \mu \mathrm{m}$ ) 

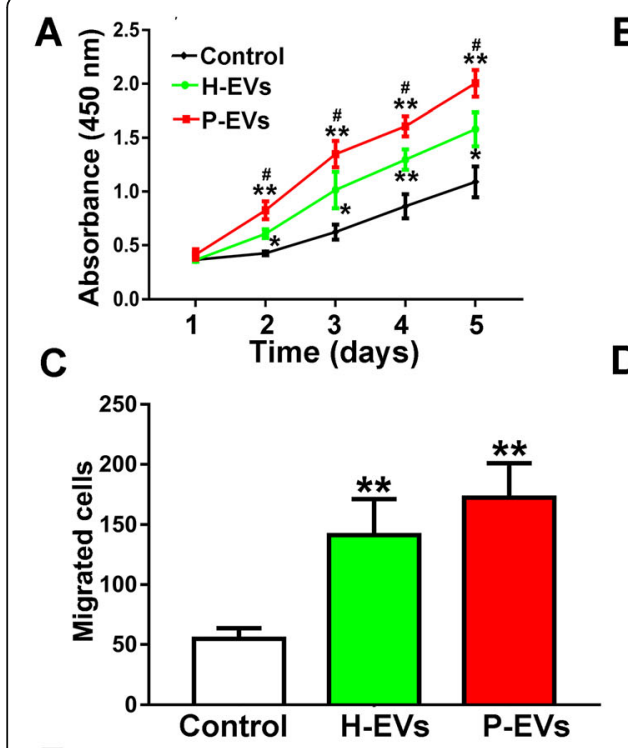

E

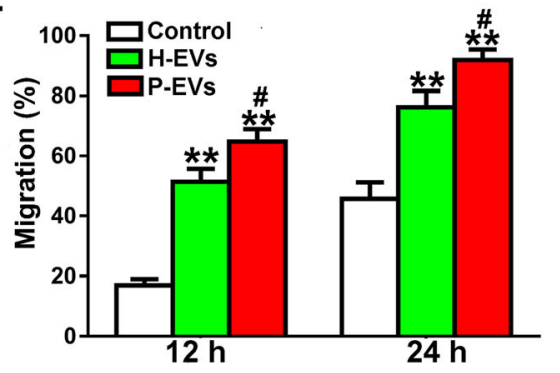

B

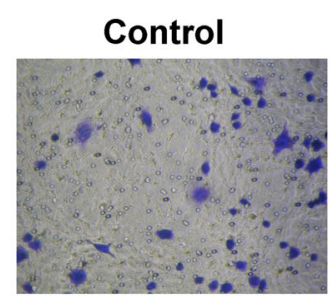

$\mathbf{O h}$
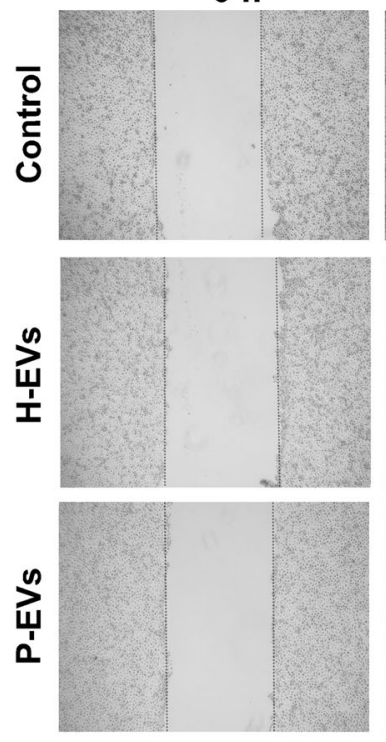

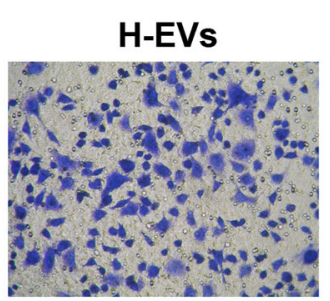

$12 \mathrm{~h}$
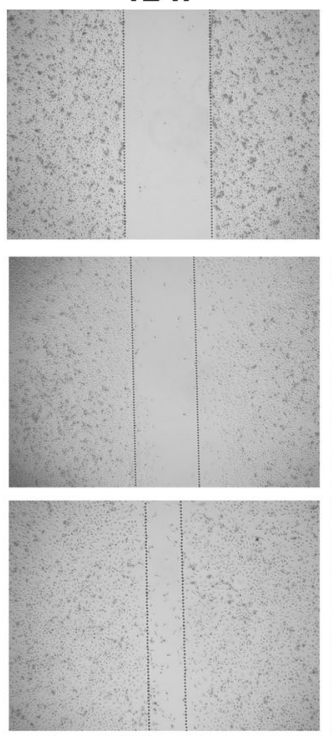

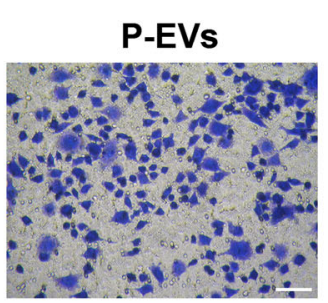

$24 \mathrm{~h}$
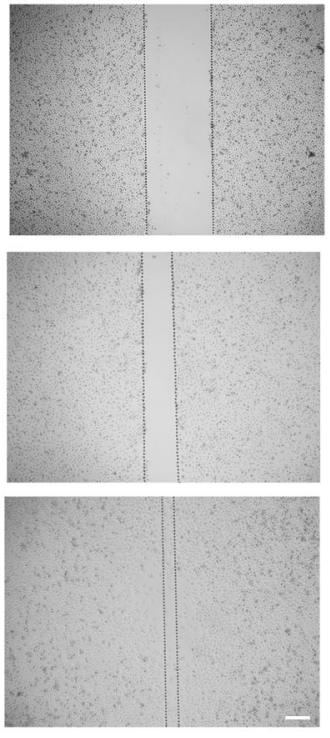

Fig. 5 EVs enhanced the proliferation and migration of ECs in vitro. a The proliferation of ECs exposed to H-EVs, P-EVs, and an equal volume of PBS was tested by the CCK-8 assay $(n=3)$. b The migration of ECs stimulated by H-EVs, P-EVs, and PBS was detected by transwell assay (scale bar, $100 \mu \mathrm{m})$. c Quantitative analysis of the migrated cells in $\mathbf{b}(n=5)$. $\mathbf{d}$ Representative images of the scratch wound assay in ECs treated with $\mathrm{H}-\mathrm{EV}$, P-EVs, and PBS (scale bar, $200 \mu \mathrm{m})$. e Quantitative analysis of the migration rates in $\mathbf{d}(n=3)$. ${ }^{*} P<0.05$, ${ }^{*} P<0.01$ vs. the control group; ${ }^{\#} P<0.05$ vs. the H-EV group

pulp tissue is critically limited [30]. Given the potential of DPSCs to differentiate into ECs and to promote angiogenesis, current bioengineering approaches have focused on the use of those cells for dental pulp tissue engineering with the aim of regenerating vascularized pulp tissues [31-33]. As a more readily available cell source in the clinic, P-DPSCs exhibit similar regenerative potential to H-DPSCs and have also been shown to give rise to functional pulp regeneration [11-15]. However, the proangiogenic capacity of P-DPSCs has not yet been described. This is the first study to explore the role of PDPSCs in angiogenesis/vasculogenesis, and our findings will help pave the way to using this easily assessable cell population in dental pulp tissue engineering.

With in-depth studies of MSCs, growing evidence has demonstrated that the therapeutic effects of MSCs contributing to angiogenesis and tissue repair are predominantly attributed to paracrine function [34]. These paracrine secretions include a variety of soluble factors and EVs, which regulate repair and regeneration processes by affecting the activity of resident cells $[35,36]$. EVs, in particular, have been confirmed as the principal factor contributing to the paracrine function-mediated therapeutic efficacy of donor cells [37]. These nanosized EVs are lipid bilayer structures containing proteins, nucleic acids, and lipids that can contribute to intercellular communications by transferring these specific molecules, further exerting biological effects $[38,39]$. In fact, EVs are attractive as therapeutics due to their cellular-related advantages, such as high stability, no risk of aneuploidy, and low propensity to trigger immune rejection following allogeneic administration [40-42]. In this context, an increasing number of studies have demonstrated that EVs derived from various types of stem cells have therapeutic potential for angiogenesis [43-45]. More importantly, EVs derived from normal DPSCs have been demonstrated to trigger regeneration of dental pulp-like tissue [18] and indeed exhibit the capacity to promote angiogenesis in vitro and in vivo [19]. Therefore, the secreted elements contained within CMs derived from P-DPSCs, particularly their secreted EVs, were applied to investigate the proangiogenic effects of P-DPSCs. 


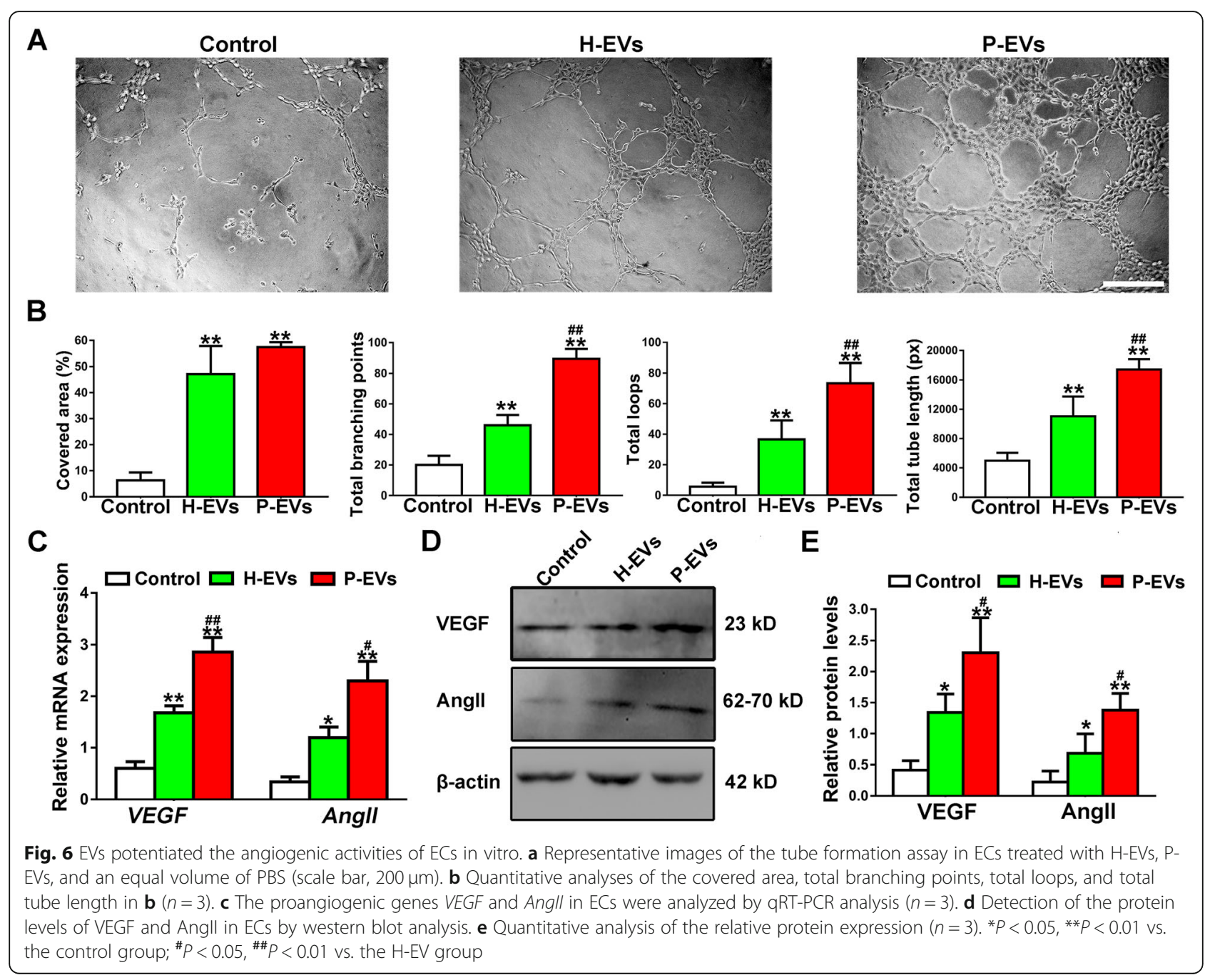

In this particular study, patient-matched H-DPSCs/EVs were used as the control for P-DPSCs/EVs to eliminate the interference of some confounding factors (e.g., age and gender). We confirmed the proangiogenic effects of P-EVs and, for the first time, to the best of our knowledge, compared their proangiogenic potential with that of $\mathrm{H}-$ EVs. Although both H-EVs and P-EVs were found to enhance the angiogenesis-related activities of ECs, P-EVs exerted a more robust potential to stimulate EC proliferation, migration, and tube formation (Figs. 5 and 6). Additionally, a recent study demonstrated that EVs derived from lipopolysaccharide (LPS)-preconditioned DPSCs had a better ability to promote Schwann cell proliferation, migration, and odontogenic differentiation than EVs from normal DPSCs [46]. In addition, MSCs were reported to secrete more EVs under inflammatory conditions than under normal conditions [47]. In line with this, P-DPSCs secreted more EVs than H-DPSCs (P-EVs vs. H-EVs, $1.73 \times 10^{9}$ vs. $5.67 \times 10^{8}$ particles $/ \mathrm{mL}$ ) in the present study. When a full-thickness skin defect model of C57BL/6 mice was applied to explore the effects of EVs on angiogenesis, both P-EVs and H-EVs were found to accelerate wound healing and promote vascularization across skin defects in mice, but wounds treated with P-EVs resulted in a quicker healing outcome and led to more new vessel formation (Figs. 7 and 8). These encouraging results collectively suggest that P-EVs possess a relatively higher proangiogenic ability than H-EVs. As reported previously, a fullthickness skin defect in mice is a valid animal model to investigate the initial proangiogenic effects of cellular materials in terms of vascularization and new vessel formation [21, 23]. However, the potential use of P-DPSCs/PEVs toward regenerating vascularized pulpal tissues must be tested in relevant pulp insult animal models.

Although our findings suggest that the inflammatory microenvironment in periodontitis does not negatively affect the proangiogenic effects of P-DPSCs, those cells were found to express lower levels of CD146 than $\mathrm{H}$ DPSCs isolated from the pulp tissue of the same donor (Fig. 1), suggesting that the cells must have undergone 

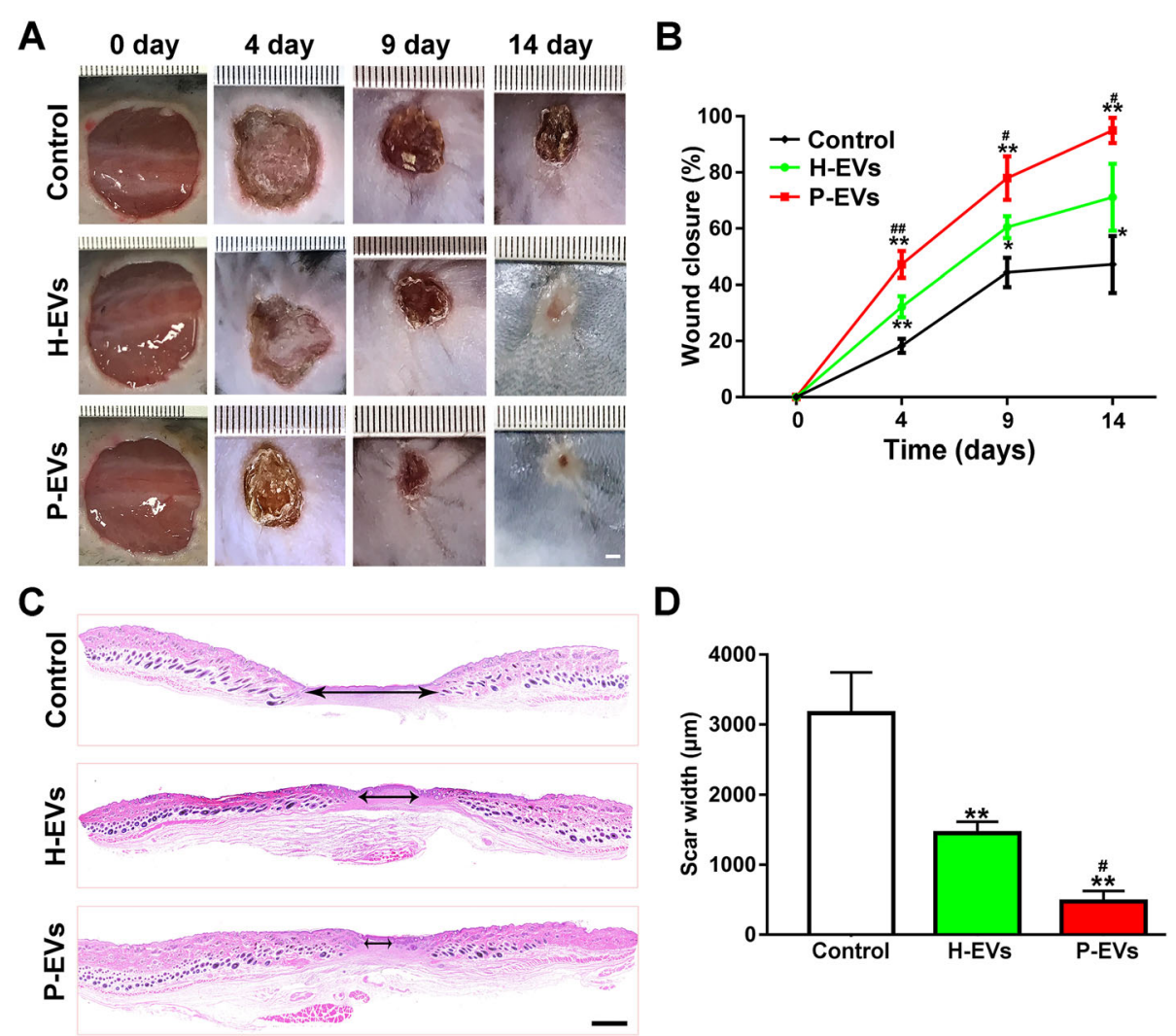

Fig. 7 EVs accelerated cutaneous wound healing in mice. a Gross view of wounds treated with H-EVs, P-EVs, and PBS at days 4, 9, and 14 postwounding (scale bar, $1.5 \mathrm{~mm})$. $\mathbf{b}$ The rate of wound closure in wounds receiving different treatments at the indicated times $(n=10)$. $\mathbf{c}$ H\&E staining of wound sections treated with $\mathrm{H}-\mathrm{EV}$ s, P-EVs, and PBS at 14 days after operation. The double-headed black arrows indicate the edges of the scars (scale bar, $500 \mu \mathrm{m}$ ). d Quantitative analysis of scar widths in $\mathbf{c}(n=8)$. ${ }^{*} P<0.05$, ${ }^{*} P<0.01$ vs. the control group; ${ }^{\#} P<0.05,{ }^{\# \#} P<0.01$ vs. the H-EV group

some changes in response to inflammation $[48,49]$. Indeed, the periodontium closely connects to the pulpdentin complex by three interconnected routes, namely, the apical foramen, the dentinal tubules, and the lateral canals, which means that once periodontitis involves a certain tooth, the dental pulp may be negatively affected by bacteria, especially by their byproducts residing in the periodontitis pocket $[50,51]$. For example, LPS is produced by gram-negative bacteria, which can induce various types of proinflammatory cytokines and chemokines such as IL-1, IL-6, and IL-8 as well as tumor necrosis factor alpha (TNF-a); these proinflammatory cytokines may affect a series of biological processes such as survival, proliferation, and differentiation of MSCs [11, 52-56]. In addition, the pulp is tightly enclosed by dentin (hard tissues), and the apical foramen is almost the only root to supply blood for pulp tissues. Therefore, if periodontal inflammation cannot be arrested, combined with poor blood flow, the dental pulp will inevitably undergo necrosis [52]. However, in our previous study, it was demonstrated that although P-DPSCs suffer relatively long-term stimulation by inflammation, regenerativerelated properties such as osteoblastic differentiation might increase to some extent, as long as the vitality of the pulp has not been totally damaged [14]. Herein, in the present study, we ruled out teeth with completely necrotized pulp tissue before pulling them out. As long as the pulp is still viable, to counteract the inflammatory invasion, P-DPSCs may have a specific capacity for the production of proangiogenic molecules, such as VEGF, because angiogenesis could be induced by inflammation $[11,52,57]$. Generally, the formation of new blood vessels is in direct response to tissue demands [58]. Thus, PDPSCs in turn strongly produce VEGF in response to diverse stimulants, and new vessels appear in the inflammatory sites of dental pulp to compensate for the insufficient blood supply in the pulp cavity.

MSC-derived EVs can load certain molecules that recapitulate the biological effects of parent cells [59]. Indeed, EVs from different stem cells possess innate differences, further leading to diverse biological functions, as they faithfully reflect the genomic characteristics of their source cells [40]. Thus, P-EVs may contain certain proangiogenic molecules that can mediate angiogenesis activities. However, the underlying mechanisms by which P-EVs promote angiogenesis remain unclear. It 


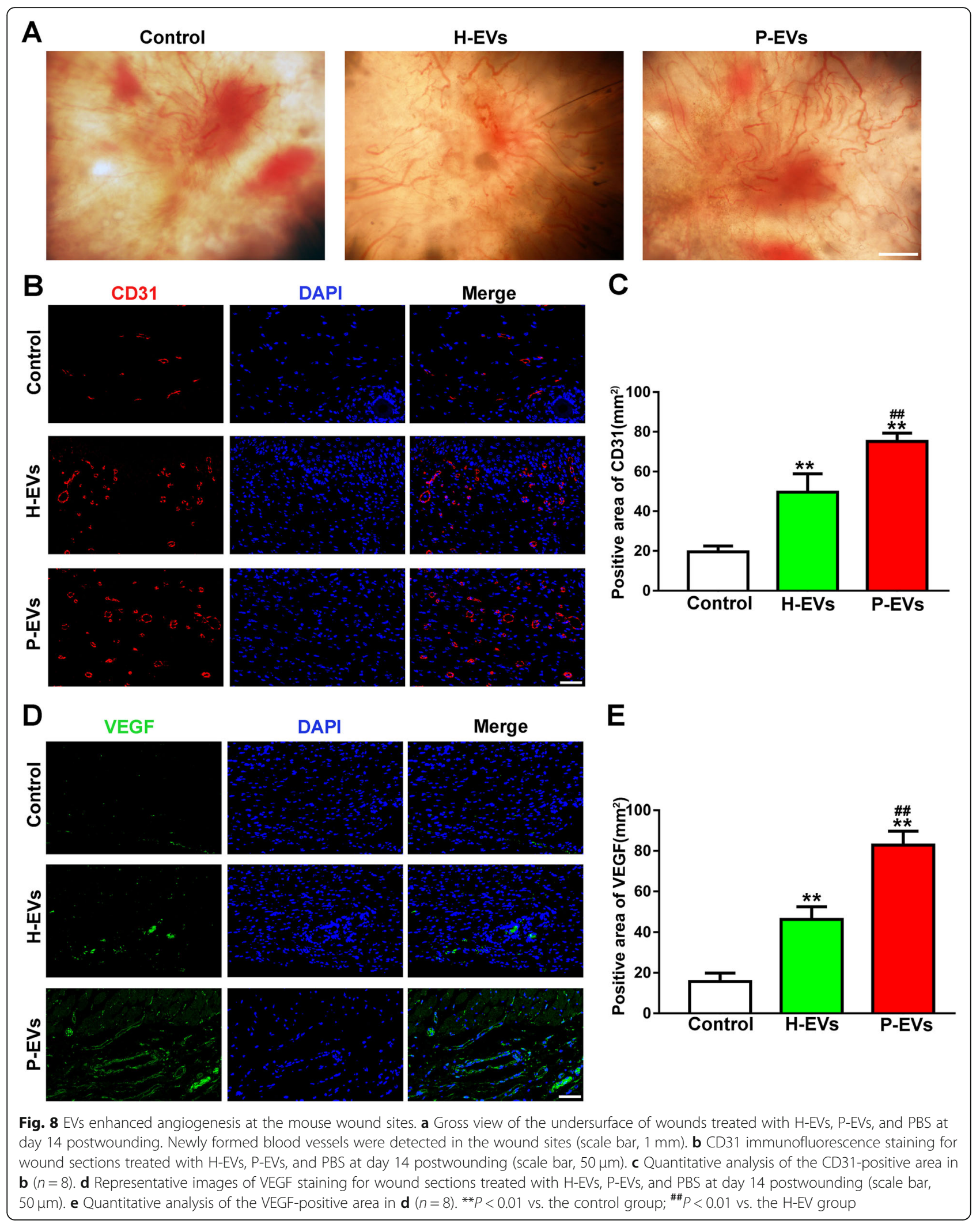


has been reported that EVs derived from MSCs contain various proteins and microRNAs that can mediate the bioactivity of target cells via activation of certain signaling pathways as well as regulation of related protein translation $[60,61]$. Thus, in the next study, we will compare the composition of biomolecules (such as common or cell-specific microRNA) in P-EVs and H-EVs to explore the underlying mechanisms by which P-EVs enhance angiogenic activities. Such investigations may provide more specific information to help identify the effect of periodontitis on pulpal property changes and the proper use of P-DPSCs in regenerative medicine.

\section{Conclusions}

Based on a comparison with patient-matched H-DPSCs, this is the first endeavor to describe the proangiogenic potential of P-DPSCs with an emphasis on their secreted EVs. Data obtained from this study showed that P-EVs derived from P-DPSCs could profoundly enhance the proliferation, migration, and angiogenesis of ECs in vitro and were able to accelerate cutaneous wound healing and promote vessel formation in vivo. Our findings suggest that the use of P-DPSCs can facilitate new vessel formation in cellular therapy and regenerative medicine. More importantly, our data reveal that although the inflammatory microenvironment across the periodontium can negatively influence the living condition of dental pulp and, hence, impair the pluripotential capacity of residing DPSCs, the proangiogenic potential of those cells is not affected. In contrast, living in an environment with the presence of various proinflammatory cytokines (e.g., slightly inflamed pulp tissue as a result of periodontitis) may enhance the proangiogenic potential of P-DPSCs. Although the present study further explores the potential to use P-DPSCs in dental pulp tissue engineering, the molecular mechanism underlying the enhanced proangiogenic effect of those cells remains unknown. In particular, the signaling pathways involved in EVs derived from P-DPSCs during angiogenesis/vasculogenesis warrant further investigation.

\section{Supplementary information}

Supplementary information accompanies this paper at https://doi.org/10. 1186/s13287-020-01614-w.

Additional file 1: Figure S1. Isolation of H-DPSCs and I-DPSCs. (A) Representative images of primary cells derived from human pulp tissue within periodontally healthy teeth (upper) and periodontally compromised teeth (lower) at day 7 (scale bar: $500 \mu \mathrm{m}$ ). (B) Morphologic appearance of H-DPSCs and P-DPSCs observed by an inverted microscope (Scale bar: $500 \mu \mathrm{m})$.

Additional file 2: Figure S2. GW4869 inhibited EV secretion without influencing cell growth. DPSCs were treated for $12 \mathrm{~h}$ with GW4869 $(10 \mu \mathrm{M})$ or DMSO. Then, the cells were washed with PBS and cultured with serum-free a-MEM media. (A) After further incubation for $48 \mathrm{~h}$, the number of cells was counted at the end of EV production. (B) The EVs were purified, and the total protein of EVs was detected $(n=3)$. ${ }^{*} P<0.01$ vs. DMSO group.

\section{Abbreviations}

MSC: Mesenchymal stem cell; DPSCs: Dental pulp stem cells; H-DPSCs: Dental pulp stem cells within healthy teeth; P-DPSCs: Dental pulp stem cells from teeth extracted due to periodontitis; ECs: Endothelial cells; CM: Conditioned medium; EV: Extracellular vesicle; H-EVs: Extracellular vesicles from dental pulp stem cells within healthy teeth; P-EVs: Extracellular vesicles from dental pulp stem cells within teeth extracted due to periodontitis; CCK-8: Cell Counting Kit-8; EGM-2: Endothelial growth medium-2; qRT-PCR: Quantitative reverse transcriptase-polymerase chain reaction; H\&E: Hematoxylin and eosin

\section{Acknowledgements}

We acknowledge that the cell-based research of this study was performed at Shaanxi Provincial Key Laboratory of Free Radical Biology and Medicine,

School of Public Health, FMMU.

\section{Authors' contributions}

$H Z, X L, Y A$, and B-MT contributed to the conception and design of the study. $H Z, X L, Y Y$, and $X-T H$ acquired, analyzed, and interpreted the data and wrote the manuscript. Y-LH, L-AW, and F-MC contributed to the study conception and design, provided financial support, and helped write the manuscript. All authors read and approved the final manuscript.

\section{Funding}

This study was supported by the National Natural Science Foundation of China (81970947, 81530050, 81771095, 81700971 and 81800971), the Shaanxi Key Scientific and Technological Innovation Team (2017KCT-32), and the Changjiang Scholars Program of the Ministry of Education of the People's Republic of China (2016).

\section{Availability of data and materials}

All data generated or analyzed during this study are included in this published article.

\section{Ethics approval and consent to participate}

The experimental protocol of this study was approved by the Ethics Committee of the Stomatological Hospital of FMMU (201203), and informed consent was signed by all the subjects who donated their extracted teeth for cell isolation.

\section{Consent for publication}

Not applicable.

\section{Competing interests}

The authors declare that they have no competing interests.

Received: 12 January 2020 Revised: 16 February 2020

Accepted: 20 February 2020 Published online: 06 March 2020

\section{References}

1. Botelho J, Cavacas MA, Machado V, Mendes JJ. Dental stem cells: recent progresses in tissue engineering and regenerative medicine. Ann Med. 2017;49:644-51.

2. Piva E, Tarle SA, Nor JE, Zou D, Hatfield E, Guinn T, et al. Dental pulp tissue regeneration using dental pulp stem cells isolated and expanded in human serum. J Endod. 2017:43:568-74.

3. Hu J, Cao Y, Xie Y, Wang H, Fan Z, Wang J, et al. Periodontal regeneration in swine after cell injection and cell sheet transplantation of human dental pulp stem cells following good manufacturing practice. Stem Cell Res Ther. 2016;7:130.

4. d'Aquino R, De Rosa A, Lanza V, Tirino V, Laino L, Graziano A, et al. Human mandible bone defect repair by the grafting of dental pulp stem/progenitor cells and collagen sponge biocomplexes. Eur Cell Mater. 2009;18:75-83.

5. Mead B, Logan A, Berry M, Leadbeater W, Scheven BA. Concise review: dental pulp stem cells: a novel cell therapy for retinal and central nervous system repair. Stem Cells. 2017;35:61-7.

6. Alge DL, Zhou D, Adams LL, Wyss BK, Shadday MD, Woods EJ, et al. Donor-matched comparison of dental pulp stem cells and bone marrow-derived mesenchymal stem cells in a rat model. J Tissue Eng Regen Med. 2010;4:73-81. 
7. Zhang W, Walboomers XF, Shi S, Fan M, Jansen JA. Multilineage differentiation potential of stem cells derived from human dental pulp after cryopreservation. Tissue Eng. 2006;12:2813-23.

8. Ma L, Hu J, Cao Y, Xie Y, Wang H, Fan Z, et al. Maintained properties of aged dental pulp stem cells for superior periodontal tissue regeneration. Aging Dis. 2019;10:793-806.

9. Barrachina L, Cequier A, Romero A, Vitoria A, Zaragoza P, Vázquez FJ, et al. Allo-antibody production after intraarticular administration of mesenchymal stem cells (MSCs) in an equine osteoarthritis model: effect of repeated administration, MSC inflammatory stimulation, and equine leukocyte antigen (ELA) compatibility. Stem Cell Res Ther. 2020;11:52.

10. Huang AH, Chen YK, Chan AW, Shieh TY, Lin LM. Isolation and characterization of human dental pulp stem/stromal cells from nonextracted crown-fractured teeth requiring root canal therapy. J Endod. 2009:35:673-81.

11. Alongi DJ, Yamaza T, Song Y, Fouad AF, Romberg EE, Shi S, et al. Stem/ progenitor cells from inflamed human dental pulp retain tissue regeneration potential. Regen Med. 2010;5:617-31.

12. Wang Z, Pan J, Wright JT, Bencharit S, Zhang S, Everett ET, et al. Putative stem cells in human dental pulp with irreversible pulpitis: an exploratory study. J Endod. 2010:36:820-5.

13. Werle SB, Lindemann D, Steffens D, Demarco FF, de Araujo FB, Pranke P, et al. Carious deciduous teeth are a potential source for dental pulp stem cells. Clin Oral Investig. 2016;20:75-81.

14. Sun HH, Chen B, Zhu QL, Kong H, Li QH, Gao LN, et al. Investigation of dental pulp stem cells isolated from discarded human teeth extracted due to aggressive periodontitis. Biomaterials. 2014;35:9459-72.

15. Tomasello L, Mauceri R, Coppola A, Pitrone M, Pizzo G, Campisi G, et al. Mesenchymal stem cells derived from inflamed dental pulpal and gingival tissue: a potential application for bone formation. Stem Cell Res Ther. 2017; $8: 179$

16. Radosinska J, Bartekova M. Therapeutic potential of hematopoietic stem cell-derived exosomes in cardiovascular disease. Adv Exp Med Biol. 2017; 998:221-35.

17. Rosca AM, Rayia DM, Tutuianu R. Emerging role of stem cells - derived exosomes as valuable tools for cardiovascular therapy. Curr Stem Cell Res Ther. 2017;12:134-8.

18. Huang CC, Narayanan R, Alapati S, Ravindran S. Exosomes as biomimetic tools for stem cell differentiation: applications in dental pulp tissue regeneration. Biomaterials. 2016;111:103-15.

19. Gonzalez-King H, Garcia NA, Ontoria-Oviedo I, Ciria M, Montero JA, Sepulveda P. Hypoxia inducible factor-1alpha potentiates jagged 1mediated angiogenesis by mesenchymal stem cell-derived exosomes. Stem Cells. 2017:35:1747-59.

20. Gharaei MA, Xue Y, Mustafa K, Lie SA, Fristad I. Human dental pulp stromal cell conditioned medium alters endothelial cell behavior. Stem Cell Res Ther. 2018;9:69.

21. Chen CY, Rao SS, Ren L, Hu XK, Tan YJ, Hu Y, et al. Exosomal DMBT1 from human urine-derived stem cells facilitates diabetic wound repair by promoting angiogenesis. Theranostics. 2018;8:1607-23.

22. Thery C, Amigorena S, Raposo G, Clayton A. Isolation and characterization of exosomes from cell culture supernatants and biological fluids. Curr Protoc Cell Biol. 2006; Chapter 3:Unit 3.22

23. Hu Y, Rao SS, Wang ZX, Cao J, Tan YJ, Luo J, et al. Exosomes from human umbilical cord blood accelerate cutaneous wound healing through miR-213p-mediated promotion of angiogenesis and fibroblast function. Theranostics. 2018:8:169-84.

24. Hayes $\mathrm{H}$ Jr. A review of modern concepts of healing of cutaneous wounds. J Dermatol Surg Oncol. 1977;3:188-93.

25. Zhang J, Guan J, Niu X, Hu G, Guo S, Li Q, et al. Exosomes released from human induced pluripotent stem cells-derived MSCs facilitate cutaneous wound healing by promoting collagen synthesis and angiogenesis. J Transl Med. 2015;13:49.

26. Gnecchi M, He H, Liang OD, Melo LG, Morello F, Mu H, et al. Paracrine action accounts for marked protection of ischemic heart by Akt-modified mesenchymal stem cells. Nat Med. 2005;11:367-8.

27. Bruno S, Camussi G. Role of mesenchymal stem cell-derived microvesicles in tissue repair. Pediatr Nephrol. 2013;28:2249-54.

28. Trajkovic K, Hsu C, Chiantia S, Rajendran L, Wenzel D, Wieland F, et al. Ceramide triggers budding of exosome vesicles into multivesicular endosomes. Science. 2008;319:1244-7.
29. Aranha AM, Zhang Z, Neiva KG, Costa CA, Hebling J, Nor JE. Hypoxia enhances the angiogenic potential of human dental pulp cells. J Endod. 2010;36:1633-7.

30. Nakashima M, lohara K. Regeneration of dental pulp by stem cells. Adv Dent Res. 2011;23:313-9.

31. Tran-Hung L, Mathieu S, About I. Role of human pulp fibroblasts in angiogenesis. J Dent Res. 2006;85:819-23.

32. Nakashima M, lohara K, Sugiyama M. Human dental pulp stem cells with highly angiogenic and neurogenic potential for possible use in pulp regeneration. Cytokine Growth Factor Rev. 2009;20:435-40.

33. Hilkens P, Fanton Y, Martens W, Gervois P, Struys T, Politis C, et al. Proangiogenic impact of dental stem cells in vitro and in vivo. Stem Cell Res. 2014:12:778-90.

34. Zhang K, Zhao X, Chen X, Wei Y, Du W, Wang Y, et al. Enhanced therapeutic effects of mesenchymal stem cell-derived exosomes with an injectable hydrogel for hindlimb ischemia treatment. ACS Appl Mater Interfaces. 2018;10:30081-91.

35. Meirelles LS, Fontes AM, Covas DT, Caplan Al. Mechanisms involved in the therapeutic properties of mesenchymal stem cells. Cytokine Growth Factor Rev. 2009;20:419-27.

36. Phinney DG, Pittenger MF. Concise review: MSC-derived exosomes for cellfree therapy. Stem Cells. 2017;35:851-8.

37. Hong P, Yang H, Wu Y, Li K, Tang Z. The functions and clinical application potential of exosomes derived from adipose mesenchymal stem cells: a comprehensive review. Stem Cell Res Ther. 2019;10:242.

38. Mathew SA, Naik C, Cahill PA, Bhonde RR. Placental mesenchymal stromal cells as an alternative tool for therapeutic angiogenesis. Cell Mol Life Sci. 2020;77:253-65.

39. Mendt M, Kamerkar S, Sugimoto H, McAndrews KM, Wu CC, Gagea M, et al. Generation and testing of clinical-grade exosomes for pancreatic cancer. JCl Insight. 2018;3:99263.

40. Borger V, Bremer M, Ferrer-Tur R, Gockeln L, Stambouli O, Becic A, et al. Mesenchymal stem/stromal cell-derived extracellular vesicles and their potential as novel immunomodulatory therapeutic agents. Int J Mol Sci. 2017;18:E1450.

41. Rani S, Ryan AE, Griffin MD, Ritter T. Mesenchymal stem cell-derived extracellular vesicles: toward cell-free therapeutic applications. Mol Ther. 2015;23:812-23.

42. Yu B, Zhang X, Li X. Exosomes derived from mesenchymal stem cells. Int J Mol Sci. 2014;15:4142-57

43. Liu X, Li Q, Niu X, Hu B, Chen S, Song W, et al. Exosomes secreted from human-induced pluripotent stem cell-derived mesenchymal stem cells prevent osteonecrosis of the femoral head by promoting angiogenesis. Int J Biol Sci. 2017;13:232-44

44. Wang K, Jiang Z, Webster KA, Chen J, Hu H, Zhou Y, et al. Enhanced cardioprotection by human endometrium mesenchymal stem cells driven by exosomal microRNA-21. Stem Cells Transl Med. 2017;6:209-22.

45. Ren S, Chen J, Duscher D, Liu Y, Guo G, Kang Y, et al. Microvesicles from human adipose stem cells promote wound healing by optimizing cellular functions via AKT and ERK signaling pathways. Stem Cell Res Ther. 2019;10:47.

46. Li J, Ju Y, Liu S, Fu Y, Zhao S. Exosomes derived from lipopolysaccharidepreconditioned human dental pulp stem cells regulate Schwann cell migration and differentiation. Connect Tissue Res. 2019:1-10. https://doi. org/10.1080/03008207.2019.1694010.

47. He M, Crow J, Roth M, Zeng Y, Godwin AK. Integrated immunoisolation and protein analysis of circulating exosomes using microfluidic technology. Lab Chip. 2014;14:3773-80

48. Viswanathan S, Shi Y, Galipeau J, Krampera M, Leblanc K, Martin I, et al. Mesenchymal stem versus stromal cells: International Society for Cell \& Gene Therapy (ISCT(R)) Mesenchymal Stromal Cell committee position statement on nomenclature. Cytotherapy. 2019;21:1019-24.

49. Liao J, Al Shahrani M, Al-Habib M, Tanaka T, Huang GT. Cells isolated from inflamed periapical tissue express mesenchymal stem cell markers and are highly osteogenic. J Endod. 2011;37:1217-24.

50. Pack AR. Periodontal considerations in endo/perio lesions. J N Z Soc Periodontol. 1999;84:9-13.

51. Bergenholtz $G$, Lindhe J. Effect of experimentally induced marginal periodontitis and periodontal scaling on the dental pulp. J Clin Periodontol. 1978:5:59-73.

52. Matsushita K, Motani R, Sakuta T, Nagaoka S, Matsuyama T, Abeyama K, et al. Lipopolysaccharide enhances the production of vascular endothelial growth factor by human pulp cells in culture. Infect Immun. 1999;67:1633-9. 
53. Feng $G$, Zheng $K$, Song $D$, Xu K, Huang D, Zhang $Y$, et al. SIRT1 was involved in TNF-alpha-promoted osteogenic differentiation of human DPSCs through Wnt/beta-catenin signal. In Vitro Cell Dev Biol Anim. 2016; 52:1001-11.

54. Carrero R, Cerrada I, Lledo E, Dopazo J, Garcia-Garcia F, Rubio MP, et al. IL1 beta induces mesenchymal stem cells migration and leucocyte chemotaxis through NF-kappaB. Stem Cell Rev Rep. 2012;8:905-16.

55. Yang X, Zhang S, Pang X, Fan M. Pro-inflammatory cytokines induce odontogenic differentiation of dental pulp-derived stem cells. J Cell Biochem. 2012;113:669-77.

56. Pricola KL, Kuhn NZ, Haleem-Smith H, Song Y, Tuan RS. Interleukin-6 maintains bone marrow-derived mesenchymal stem cell stemness by an ERK1/2-dependent mechanism. J Cell Biochem. 2009;108:577-88.

57. Carmeliet P. Mechanisms of angiogenesis and arteriogenesis. Nat Med. 2000;6:389-95

58. Risau W. Mechanisms of angiogenesis. Nature. 1997;386:671-4.

59. Lou G, Chen Z, Zheng M, Liu Y. Mesenchymal stem cell-derived exosomes as a new therapeutic strategy for liver diseases. Exp Mol Med. 2017;49:e346.

60. Zhao L, Hu C, Zhang P, Jiang H, Chen J. Genetic communication by extracellular vesicles is an important mechanism underlying stem cell-based therapy-mediated protection against acute kidney injury. Stem Cell Res Ther. 2019:10:119.

61. Rong X, Liu J, Yao X, Jiang T, Wang Y, Xie F. Human bone marrow mesenchymal stem cells-derived exosomes alleviate liver fibrosis through the Wnt/beta-catenin pathway. Stem Cell Res Ther. 2019;10:98.

\section{Publisher's Note}

Springer Nature remains neutral with regard to jurisdictional claims in published maps and institutional affiliations.

- fast, convenient online submission

- thorough peer review by experienced researchers in your field

- rapid publication on acceptance

- support for research data, including large and complex data types

- gold Open Access which fosters wider collaboration and increased citations

- maximum visibility for your research: over $100 \mathrm{M}$ website views per year

At $\mathrm{BMC}$, research is always in progress.

Learn more biomedcentral.com/submissions 